\title{
Transcriptomic analysis and competing endogenous RNA network in the human endometrium between proliferative and mid-secretory phases
}

\author{
SEONG-LAN YU ${ }^{1 *}$, TAE-HYUN KIM ${ }^{2 *}$, YOUNG-HYUN HAN ${ }^{1 *}$, YUJIN KANG $^{1}$, \\ DA-UN JEONG ${ }^{1}$, DONG CHUL LEE ${ }^{3}$, JAEKU KANG ${ }^{1,4}$ and SEOK-RAE PARK ${ }^{1,5}$ \\ ${ }^{1}$ Priority Research Center, Myunggok Medical Research Institute, College of Medicine, Konyang University; \\ ${ }^{2}$ Department of Obstetrics and Gynecology, Konyang University Hospital, Daejeon 35365; ${ }^{3}$ Personalized Genomic \\ Medicine Research Center, Korea Research Institute of Bioscience and Biotechnology (KRIBB), \\ Daejeon 34141; Departments of ${ }^{4}$ Pharmacology and ${ }^{5}$ Microbiology, College of Medicine, \\ Konyang University, Daejeon, 35365, Republic of Korea
}

Received January 18, 2021; Accepted April 8, 2021

DOI: $10.3892 / \mathrm{etm} .2021 .10092$

\begin{abstract}
Successful embryo implantation is the first step for establishing natural pregnancy and is dependent on the crosstalk between the embryo and a receptive endometrium. However, the molecular signaling events for successful embryo implantation are not entirely understood. To identify differentially expressed transcripts [long-noncoding RNAs (lncRNAs), microRNAs (miRNAs) and mRNAs] and competing endogenous RNA (ceRNA) networks associated with endometrial receptivity, the current study analyzed gene expression profiles between proliferative and mid-secretory endometria in fertile women. A total of 247 lncRNAs, 67 miRNAs and 2,154 mRNAs were identified as differentially expressed between proliferative
\end{abstract}

Correspondence to: Dr Jaeku Kang, Department of Pharmacology, College of Medicine, Konyang University, 158 Gwanjeodong-ro, Seo-gu, Daejeon 35365, Republic of Korea

E-mail: jaeku@konyang.ac.kr

Dr Seok-Rae Park, Department of Microbiology, College of Medicine, Konyang University, 158 Gwanjeodong-ro, Seo-gu, Daejeon 35365, Republic of Korea

E-mail: srpark@konyang.ac.kr

${ }^{*}$ Contributed equally

Abbreviations: ceRNA, competitive endogenous RNA; DE, differentially expressed; DEG, differentially expressed gene; FC, fold change; FPKM, fragments per kilobase of exon per million fragments mapped; GO, Gene Ontology; IPA, ingenuity pathway analysis; KEGG, Kyoto Encyclopedia of Genes and Genomes; lncRNA, long ncRNA; miRNA, microRNA; ncRNA, noncoding RNA; qPCR, quantitative PCR; smRNA, small noncoding RNA; UTR, untranslated region; WOI, window of implantation

Key words: proliferative endometrium, mid-secretory endometrium, endometrial receptivity, RNA sequencing, competing endogenous RNA network and mid-secretory endometria. Kyoto Encyclopedia of Genes and Genomes pathway analysis indicated that these differentially expressed genes were significantly enriched for 'cell adhesion molecules.' Additionally, 98 common mRNAs were significantly involved in tryptophan metabolism, metabolic pathways and FoxO signaling. From the differentially expressed lncRNA/miRNA/mRNA ceRNA network, hub RNAs that formed three axes were identified: The DLX6-AS1/miR-141 or miR-200a/OLFM1 axis, the WDFY3-AS2/miR-135a or miR-183/STC1 axis, and the LINC00240/miR-182/NDRG1 axis. These may serve important roles in the regulation of endometrial receptivity. The hub network of the current study may be developed as a candidate marker for endometrial receptivity.

\section{Introduction}

Successful embryo implantation, in which the blastocyst adheres to and invades the maternal endometrium, is the first step for establishing natural pregnancy. In the human endometrium, implantation occurs between 6 and 10 days after ovulation [the window of implantation (WOI)] (1), during which the human endometrium undergoes morphological and biochemical changes, including modulation of estrogen, progesterone, adhesion molecules, growth factors, cytokines, and chemokines, to facilitate implantation (2). This process enhances endometrial receptivity by balancing the expression of adhesion molecules and inhibitory proteins acting as a barrier to blastocyst implantation (3). However, implantation failure (i.e., poor endometrial receptivity) remains a serious barrier to both spontaneous and assisted pregnancies (4). To characterize the molecular mechanisms associated with endometrial receptivity, studies have identified, using microarrays or RNA sequencing (RNA-seq), hundreds of differentially expressed genes (DEGs) between prereceptive and receptive endometria (5-12). Although these studies have verified many differentially expressed (DE) transcripts, the overlap between DEGs in different studies is low, and the molecular mechanisms underlying endometrial receptivity remain unclear (13). 
Only $2 \%$ of the human transcriptome is translated into proteins; the remaining untranslated transcriptome contains noncoding RNAs (ncRNAs), which have been reported to control cellular processes and functions by regulating the expression of target genes (14). Long ncRNAs (lncRNAs) are over 200 nucleotides in length and display differential patterns of expression in various tissues, where they play diverse roles in various physiological processes (15). Additionally, lncRNAs can be used as biomarkers of implantation failure (16-18). MicroRNAs (miRNAs) are evolutionarily conserved ncRNAs, measuring 19-22 nucleotides in length, and negatively regulate target gene expression by binding to the 3'-untranslated region (UTR) of the target gene (19). Approximately half of all genes in the genome are miRNA targets regulated at the post-transcriptional level (20), and recent studies have linked miRNAs to human endometrial diseases such as endometriosis and endometrial cancer (21-23).

The competitive endogenous RNA (ceRNA) hypothesis was first proposed by Salmena et al, who demonstrated that lncRNAs can regulate other RNA transcripts by competing for target miRNAs via binding sites known as miRNA response elements (24). Since then, ceRNA networks have been shown to have roles in the development of various tumors.

In this study, we attempted to identify DE lncRNAs, miRNAs, and mRNAs associated with endometrial receptivity using RNA-seq of proliferative and mid-secretory endometrium samples. Additionally, we performed in silico analysis of genes previously reported to show differential expression between prereceptive and receptive endometrium samples. We also constructed a ceRNA network based on DE miRNA/lncRNA and miRNA/mRNA pairs as correlations between mRNAs and ncRNAs can indicate complex gene regulatory events. The hub ncRNAs from this network can be developed as candidate markers for endometrial receptivity.

\section{Materials and methods}

Endometrial tissue collection. Endometrial samples were obtained from 30 fertile women who attended Konyang University Hospital (Daejeon, Republic of Korea) and had self-reported regular, normal (21-35 days) menstrual cycles. Each woman had at least one live birth, fewer than two spontaneous abortions, and had received no medication, including hormonal treatments, for at least three months prior to the day of the biopsy. Endometrial tissues were collected during the proliferative or secretory phase of the menstrual cycle, which donors were divided with 2 groups according to the phase of menstrual period on the day of endometrial biopsy. Before the biopsy, a gynecologist checked each donor's menstrual period by checking last menstrual day and endometrial ultrasound measurements. For endometrial sampling, a sterile speculum was inserted into the vagina, a betadine dressing was applied, and endometrial tissue was gently collected using a disposable uterine sampler (Rampipella, RI.MOS, Mirandola, Italy). Each endometrial sample was divided into two tubes; one was sent to the pathologist to identify endometrial pathology and confirm the menstrual cycle by histological examination using Noyes criteria (25), and the other was used for RNA extraction. The average age and body index were not different between 2 groups $(37.5 \pm 2.7$ vs. $36.9 \pm 2.4$ years, $22.6 \pm 3.3$ vs
$22.2 \pm 3.4 \mathrm{~kg} / \mathrm{m}^{2}$ ). And the number of previous live birth or abortion history was not also different (Table I). Continuous variables were compared with the two-sample $t$-test. And categorical variables were analyzed with Fisher's exact test. All P-values were 2-sided. P-values less than 0.05 were deemed statistically significant.

Ethical approval. This study was approved by the Bioethics Committee of Konyang University Hospital (IRB File No. 2018-11-007-005). Signed informed consent was obtained from each patient.

RNA extraction. Total RNA was isolated from endometrial tissue immediately after biopsy using TRIzol reagent (Invitrogen, Carlsbad, CA, USA) according to the manufacturer's instructions. Total RNA concentrations were calculated using Quant-IT RiboGreen (Invitrogen), and integrity was assessed using a TapeStation RNA ScreenTape. Only high-quality RNA preparations with an RNA integrity number greater than 6.5 were used to construct the RNA library with total RNA isolated from four proliferative and four mid-secretory endometrium samples.

RNA library construction. Ten nanograms of RNA isolated from each sample was used to construct miRNA sequencing libraries using a SMARTer small noncoding RNA (smRNA)-Seq Kit (Takara Bio Inc.) according to the manufacturer's instructions. cDNA synthesis was primed using a $3^{\prime}$ smRNA dT primer, which incorporates an adapter sequence at the $5^{\prime}$ end of each RNA template and adds non-template nucleotides bound to oligo-enhanced SMRT smRNA with locked nucleic acid technology for greater sensitivity. For template-switching, PrimeScript RT was used with the SMART smRNA oligo as a template to add a second adapter sequence to the $3^{\prime}$ end of each first-strand cDNA molecule. Full-length Illumina adapters were added during polymerase chain reaction (PCR) amplification, and the amplified libraries were purified to remove the fraction over $171 \mathrm{bp}$ ( $>18 \mathrm{bp}$ of cDNA plus $153 \mathrm{bp}$ of adaptors).

One microgram total RNA isolated from each sample was used to prepare a total RNA library using an Illumina TruSeq Stranded Total RNA Sample Prep Kit (Illumina). rRNA was depleted using a Ribo-Zero kit (Illumina), and the remaining RNA was purified, fragmented, and primed for cDNA synthesis. Cleaved RNA fragments were converted into first-strand cDNA using reverse transcriptase and random hexamers, followed by second-strand cDNA synthesis using DNA polymerase I, RNase H, and dUTP. The cDNA fragments then underwent end repair, single ' $A$ ' base addition, and adapter ligation. The products were purified and enriched by PCR to create the final cDNA library.

Libraries were quantified by quantitative PCR (qPCR) using KAPA library quantification kits for Illumina sequencing platforms and qualified using a TapeStation D1000 ScreenTape (Agilent Technologies).

RNA sequencing. smRNA libraries underwent 51-bp single-end sequencing using Illumina HiSeq 2500 (Illumina). Indexed cDNA libraries were subjected to paired-end (2x100 bp) sequencing using an Illumina Novaseq (Illumina). 
Table I. Characteristics of endometrium donors for RNA sequencing.

\begin{tabular}{|c|c|c|c|}
\hline Variables/Group & Proliferative phase $(n=15)$ & Secretory phase $(n=15)$ & P-value \\
\hline Age (years) & $37.5 \pm 2.7$ & $36.9 \pm 2.4$ & $0.53^{\mathrm{a}}$ \\
\hline BMI $\left(\mathrm{kg} / \mathrm{m}^{2}\right)$ & $22.6 \pm 3.3$ & $22.2 \pm 3.4$ & $0.77^{\mathrm{a}}$ \\
\hline Number of live births, n (\%) & & & $0.09^{\mathrm{b}}$ \\
\hline 1 & $4(26.6)$ & $7(46.6)$ & \\
\hline 2 & $7(46.6)$ & $8(53.3)$ & \\
\hline 3 & $4(26.6)$ & $0(0.0)$ & \\
\hline Number of abortions, $\mathrm{n}(\%)$ & & & $0.13^{\mathrm{b}}$ \\
\hline 0 & $13(86.6 \%)$ & $10(66.6 \%)$ & \\
\hline 1 & $1(6.6 \%)$ & $5(33.3 \%)$ & \\
\hline 2 & $1(6.6 \%)$ & $0(0.0 \%)$ & \\
\hline
\end{tabular}

P-values were determined using the aStudent t-test and ${ }^{\mathrm{b}}$ Fisher's exact test. Age and BMI data are presented as the mean \pm SD.

The reference genome sequence for Homo sapiens (hg19) and annotation data were downloaded from the National Center for Biotechnology Information, and known transcripts were assembled using StringTie v1.3.4d (26). Transcript abundance and gene expression were calculated as the read count or fragments per kilobase of exon per million fragments mapped (FPKM) value per sample. We deposited smRNA sequences derived from sequencing of smRNA libraries into Gene Expression Omnibus (GEO) of National Center for Biotechnology Information (NCBI) (https://www.ncbi.nlm. nih.gov/geo/query/acc.cgi?acc=GSE167325). The expression profiles were used to identify DEGs. In groups with different conditions, DEGs or transcripts were filtered by statistical hypothesis testing.

Statistical analysis of differential miRNA and total RNA expression. Raw data were normalized using the relative log expression method in DESeq2 (27). miRNAs with no count in more than $50 \%$ of samples were excluded, leaving 507 mature miRNAs for further analysis. Various plots were drawn using normalized log transformation. Two groups were compared using the 'nbinomWaldTest' in DESeq2. Relative gene abundance was measured in FPKM using StringTie. Statistical analysis was performed on the estimated abundance of each gene in the samples to identify DEGs. Genes with more than one 'zero' FPKM value in the samples were excluded. The statistical significance of the differential expression data was determined using independent $t$-tests based on fold changes and the null hypothesis that no difference existed. The false discovery rate was controlled by adjusting the $P$-value using the Benjamini-Hochberg algorithm.

Hierarchical clustering analysis. Hierarchical clustering analysis was performed using complete linkage and Euclidean distance as a measure of similarity to display the expression patterns of DE miRNAs and transcripts that satisfied fold change (FC) greater than or equal to 1.5 and a raw P-value of less than 0.05. All data analysis and DEG visualization were conducted using R v.3.6.0 (www.r-project.org).
Functional enrichment analysis. Gene ontology (GO) and Kyoto Encyclopedia of Genes and Genomes (KEGG) functional enrichment analyses of DE mRNAs were performed using the Database for Annotation, Visualization, and Integrated Discovery (https://david.ncifcrf.gov/). All DEG data analysis and visualization were conducted using R v.3.6.0 (www.r-project.org).

Reverse transcription ( $R T)$ - $q P C R$. RNA samples isolated from 14 proliferative and nine mid-secretory phase endometrium samples were subjected to RT-qPCR. To determine mRNA and lncRNA expression levels, cDNA was synthesized using M-MLV reverse transcriptase (Promega). qPCR was performed using a CFX 96 qPCR instrument and iQ SYBR Green Supermix (Bio-Rad Laboratories) with the following amplification conditions: Initial denaturation at $95^{\circ} \mathrm{C}$ for $3 \mathrm{~min}$; followed by 40 cycles of denaturation at $95^{\circ} \mathrm{C}$ for $10 \mathrm{~s}, 60^{\circ} \mathrm{C}$ for $10 \mathrm{~s}$, and extension at $72^{\circ} \mathrm{C}$ for $15 \mathrm{~s}$. We performed TaqMan microRNA assays for determination of miRNA expression levels. The primer sequences are presented in Tables SI and SII. mRNA and IncRNA expression levels were quantified following normalization to glyceraldehyde 3-phosphate dehydrogenase (GAPDH) expression and miRNA expression levels were quantified following normalization to RNA, U6 small nuclear 6 , pseudogene (RNU6B) using the 2- $\Delta \Delta \mathrm{CT}$ method, the fold change (FC) was evaluated in comparison with the proliferative phase. Assays were conducted in triplicate. The data are presented as the mean \pm standard error of the mean (SEM). The Student's t-test or Mann-Whitney U test was used to assess between group differences.

Ingenuity pathway analysis (IPA). To investigate the associated gene function networks, IPA was performed (Ingenuity Systems; http://www.ingenuity.com).

ceRNA network construction. To investigate potential interactions among lncRNAs, miRNAs, and mRNAs, we constructed a ceRNA network. Interactions between DE lncRNAs and miRNAs were predicted using miRcode (http://www.mircode. org/). Interactions between DE miRNAs and mRNAs were 


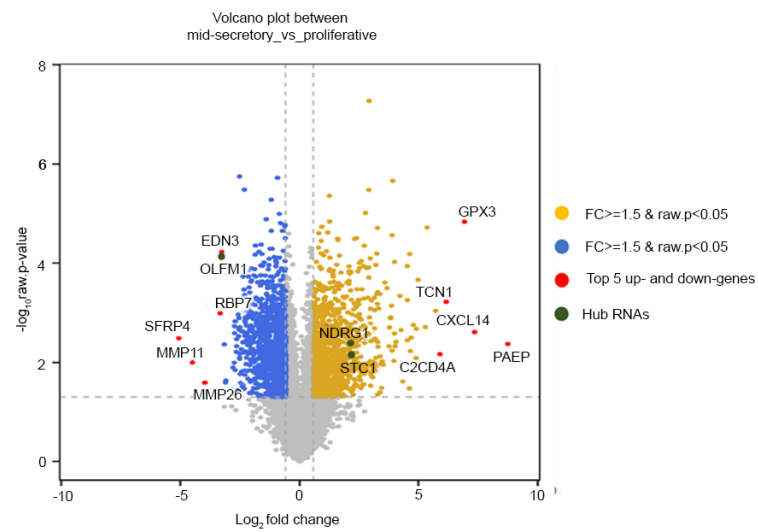

C

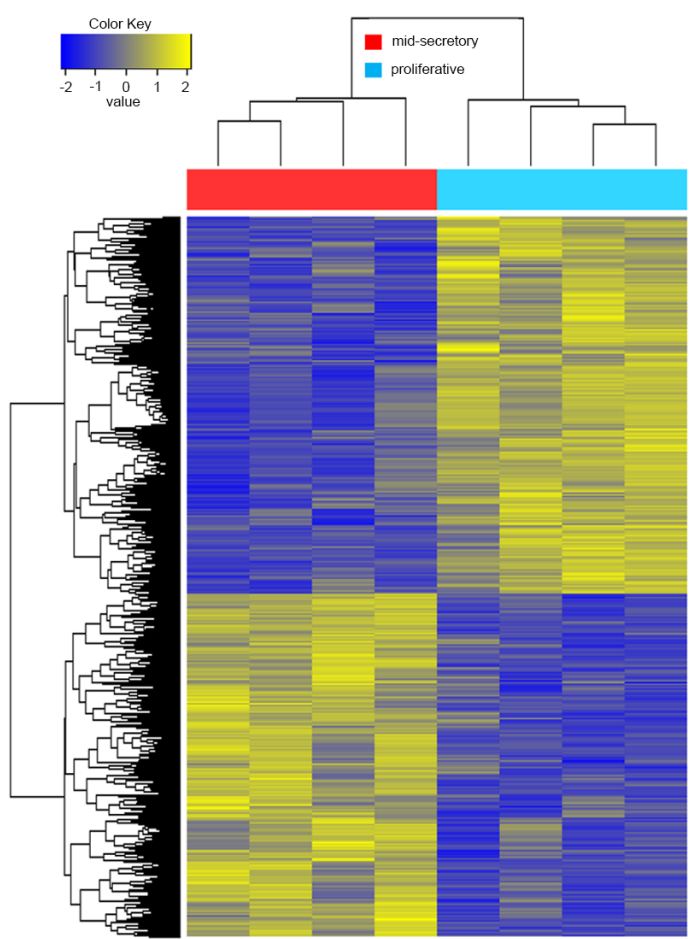

B



D

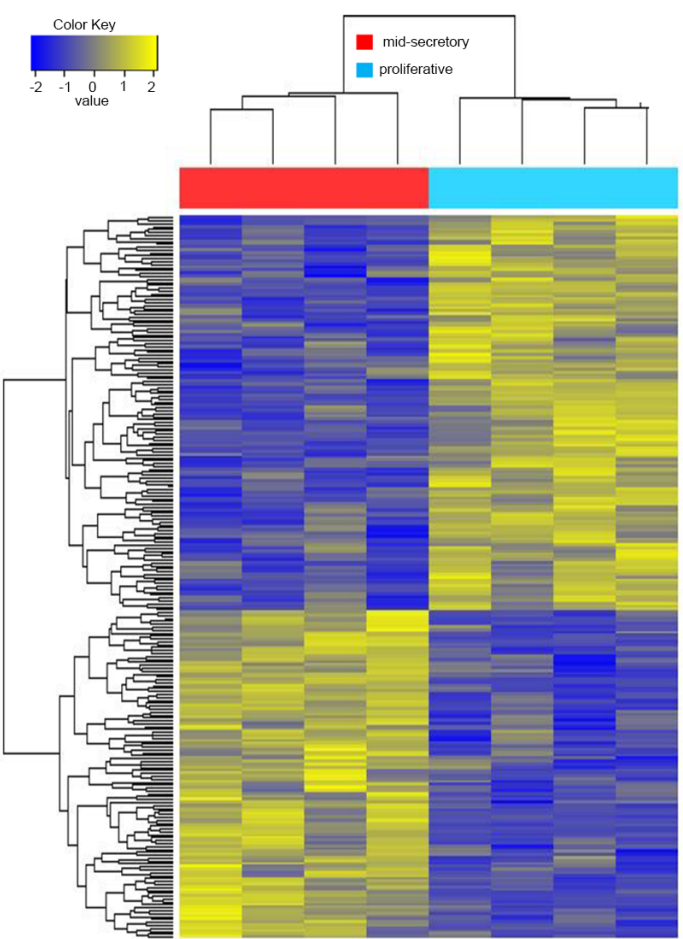

Figure 1. Volcano plots and hierarchical clustering analysis of the DE transcripts in proliferative and mid-secretory endometria. Volcano plots of DE (A) mRNAs and (B) lncRNAs. The top 5 up and down DE transcripts are indicated by red circles. The hub RNAs are indicated by green circles. Hierarchical clustering analysis of the DE (C) mRNAs and (D) lncRNAs. Transcripts were selected using $\mathrm{P}<0.05$ and an FC $\geq 1.5$ or $\leq-1.5$. Blue boxes indicate proliferative endometrium samples. Red boxes indicate mid-secretory endometrium samples. DE, differentially expressed; lncRNA, long non-coding RNAs; up, upregulated; down, downregulated; FC, fold change.

predicted using mirDIP (http://ophid.utoronto.ca/mirDIP; 'bidirectional search'). The DE lncRNA/miRNA/mRNA ceRNA network was visualized and constructed using the Cytoscape software (28).

\section{Results}

Identification of DE transcripts. To investigate genes associated with endometrial receptivity, we analyzed DE transcripts between proliferative and mid-secretory endometrium samples using next-generation sequencing (NGS). We identified 2,154 DE mRNAs, of which 1,042 (48.37\%) were upregulated and $1,112(51.63 \%)$ were downregulated in the mid-secretory endometrium (Table SIII). Variance in the DE mRNAs in the proliferative or mid-secretory endometrium, visualized using volcano plots, indicated the top 5 up- and downregulated genes (Fig. 1A). Additionally, we identified 247 significant DE IncRNAs, of which 112 (45.34\%) were upregulated and 135 $(54.66 \%)$ were downregulated in the mid-secretory endometrium (Table SIV). In Fig. 1B, a volcano plot, displaying the differences in the expression values of the DE lncRNAs in the proliferative or mid-secretory endometrium, indicated the top 5 up- and downregulated lncRNAs. The expression of DE mRNAs and lncRNAs in the proliferative or mid-secretory endometrium is illustrated in Fig. 1C and D. Notably, DE mRNAs and lncRNAs were able to distinguish between the proliferative and mid-secretory endometria. The top 10 upand downregulated DE mRNAs and lncRNAs are indicated in Tables II and III, respectively.

Identification of DE miRNAs. We next checked the expression patterns of miRNAs from NGS expression data. In 
Table II. Top 10 differentially expressed upregulated and downregulated mRNAs with the highest fold change.

A, Upregulated mRNAs

\begin{tabular}{llcc}
\hline Symbol & \multicolumn{1}{c}{ Gene name } & FC & P-value \\
\hline PAEP & Progestagen associated endometrial protein & 426.4482 & 0.0043 \\
CXCL14 & C-X-C motif chemokine ligand 14 & 162.8550 & 0.0024 \\
GPX3 & Glutathione peroxidase 3 & 121.0577 & 71.4916 \\
TCN1 & Transcobalamin 1 & 59.5485 & $6.03 \times 10^{-4}$ \\
C2CD4A & C2 calcium-dependent domain containing 4A & 52.3765 & 0.0069 \\
DPP4 & Dipeptidyl peptidase 4 & 40.8157 & $9.10 \times 10^{-4}$ \\
C4BPA & Complement component 4 binding protein alpha & 38.7763 & $1.89 \times 10^{-5}$ \\
AOX1 & Aldehyde oxidase 1 & 31.4066 & 0.0019 \\
CFD & Complement factor D & 29.9378 \\
DKK1 & Dickkopf WNT signaling pathway inhibitor 1 & $2.17 \times 10^{-4}$ \\
\hline
\end{tabular}

B, Downregulated mRNAs

\begin{tabular}{llcc}
\hline Symbol & \multicolumn{1}{c}{ Gene name } & FC & P-value \\
\hline SFRP4 & Secreted frizzled-related protein 4 & -33.4871 & 0.0033 \\
MMP11 & Matrix metallopeptidase 11 & -22.7452 & 0.0101 \\
MMP26 & Matrix metallopeptidase 26 & -15.8265 & 0.0255 \\
RBP7 & Retinol binding protein 7 & -10.1247 & 0.0010 \\
EDN3 & Endothelin 3 & -9.6811 & $5.92 \times 10^{-5}$ \\
OLFM1 & Olfactomedin 1 & -9.6540 & $7.57 \times 10^{-5}$ \\
SERPINA5 & Serpin family A member 5 & -8.9736 & 0.0043 \\
POSTN & Periostin & -8.8430 & $7.77 \times 10^{-4}$ \\
PKHD1L1 & PKHD1 like 1 & -8.6865 & 0.0254 \\
SLC47A1 & Solute carrier family 47 member 1 & -8.5516 & 0.0231 \\
\hline
\end{tabular}

FC, fold change.

total, 507 miRNAs were identified with small RNA-seq (GSE167325), of which 67 were DE in the mid-secretory endometrium compared with the proliferative endometrium. Of the 67 DE miRNAs, 32 (47.76\%) were upregulated, and 35 $(52.24 \%)$ were downregulated in the mid-secretory endometrium (Table SV). The DE miRNAs were visualized using a volcano plot, which indicated the top 5 up- and downregulated genes, and a heatmap (Fig. 2A and B). Based on the heatmap of the DE miRNAs, the mid-secretory endometrium could be clustered separately from the paired proliferative endometrium. The top ten up- and downregulated DE miRNAs are summarized in Table IV.

$G O$ and KEGG pathway analysis. To gain insights into the functions of the DE mRNAs in the proliferative and mid-secretory endometria, we performed GO and KEGG analyses of DEGs. GO classes were separated into three major categories. In the biological process category, DE mRNAs upregulated in the mid-secretory endometrium were significantly enriched in response to stimulus, localization, immune system process, and immune response (Fig. 3A), whereas downregulated DE mRNAs were significantly enriched in cell cycle and chromosome organization (Fig. 3D). In the cell component category, upregulated DE mRNAs were mainly enriched in cytoplasm, cytoplasmic part, cell periphery, and plasma membrane (Fig. 3B), whereas downregulated DE mRNAs were enriched in nonmembrane-bound organelles, intracellular nonmembrane-bound organelles, chromosomes, and chromosomal parts (Fig. 3E). In the molecular function category, upregulated DE mRNAs were dominantly enriched in protein binding (Fig. 3C), and downregulated DE mRNAs were enriched in protein binding and DNA binding (Fig. 3F).

The top 20 enriched KEGG pathways included phosphatidylinositol 3-kinase/AKT signaling, cell adhesion molecules, focal adhesion, cAMP signaling, and Rapl signaling pathways (Fig. 3G). These pathways are associated with the upregulation of essential cell adhesion-related molecules (integrin, cadherin, selectin, and laminin) in the mid-secretory endometrium (29). These results suggest that these pathways mainly contribute to the expression of adhesion molecules in endometrial epithelial cells during the receptive phase.

Validation of DE lncRNAs, miRNAs, and mRNAs. To verify the RNA sequencing results, we investigated relative ncRNA expression by RT-qPCR. The validated results of NGS suggested that LINC00598 and HAND2 antisense 
Table III. Top 10 differentially expressed upregulated and downregulated lncRNAs with the highest fold change.

A, Upregulated lncRNAs

\begin{tabular}{llcc}
\hline Transcript ID & \multicolumn{1}{c}{ Gene symbol } & FC & P-value \\
\hline NR_123740 & RIMKLB, variant3 & 30.2256 & 0.0033 \\
NR_121625 & LINC02432 & 15.5469 & $1.16 \times 10^{-4}$ \\
NR_046371 & CP, variant2 & 15.3309 & 0.0067 \\
NR_120480 & IL6ST, variant4 & 14.7984 & 0.0118 \\
NR_110114 & LOC101927668 & 7.9406 & 0.0165 \\
NR_126404 & LINC01320 & 7.8229 & 0.0019 \\
NR_045623 & NABP1, variant4 & 7.0512 & 0.0131 \\
NR_131782 & CCEPR & 6.4217 & 0.0158 \\
NR_026979 & DRAIC & 6.0252 & 0.0151 \\
NR_045580 & RXFP1, variant9 & 5.6729 & 0.0089 \\
\hline
\end{tabular}

B, Downregulated lncRNAs

\begin{tabular}{llll}
\hline Transcript ID & \multicolumn{1}{c}{ Gene symbol } & FC & P-value \\
\hline NR_133013 & MMP11, variant2 & -7.9496 & 0.0166 \\
NR_002813 & KCNIP4-IT1 & -6.8875 & 0.0098 \\
NR_121610 & BMPR1B-AS1 & -6.0480 & 0.0016 \\
NR_038861 & TUNAR, variant2 & -4.8339 & 0.0291 \\
NR_120454 & LINC02449 & -4.5053 & 0.0030 \\
NR_027349 & MIR17HG, variant2 & -4.3154 & 0.0108 \\
NR_072997 & PTK7, variant7 & -4.0389 & 0.0234 \\
NR_120409 & PCSK5, variant3 & -3.8489 & 0.0202 \\
NR_039982 & LINC00639 & -3.1286 & 0.0024 \\
NR_015448 & DLX6-AS1 & -3.0147 & $8.93 \times 10^{-4}$ \\
\hline
\end{tabular}

FC, fold change; lncRNA, long non-coding RNA.

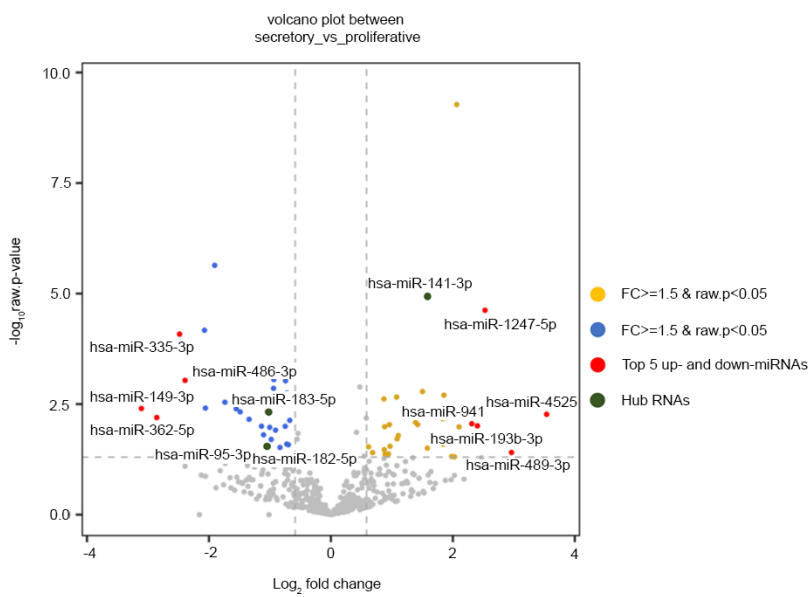

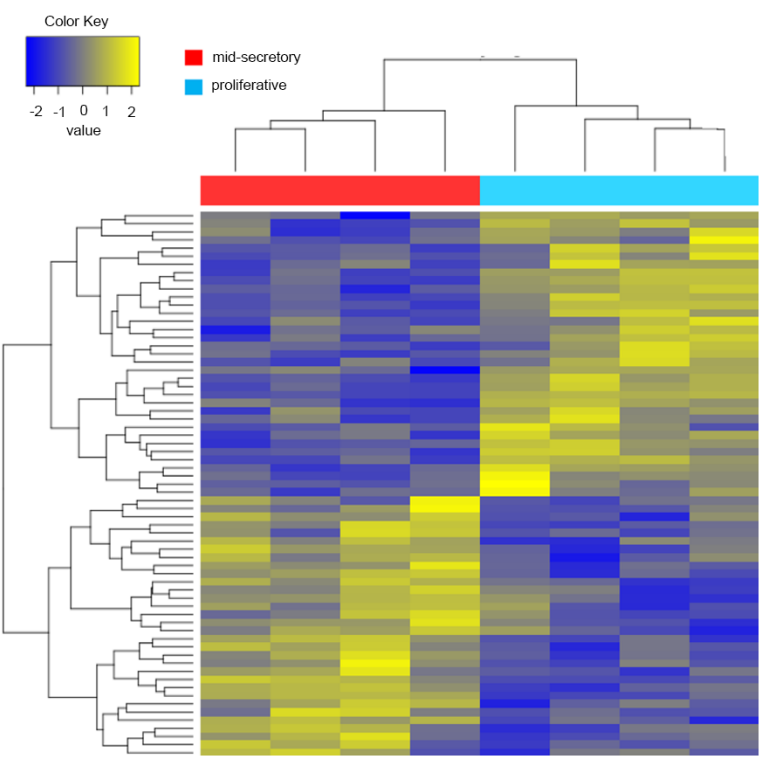

Figure 2. Volcano plot and hierarchical clustering analysis of DE miRNAs in proliferative and mid-secretory endometria. (A) Volcano plot of DE miRNAs. The top 5 up and down DE miRNAs are indicated by red circles. The hub RNAs are indicated by green circles. (B) Hierarchical clustering analysis of DE miRNAs. miRNAs were selected using $\mathrm{P}<0.05$ and $\mathrm{FC} \geq 1.5$ or $\geq-1.5$. DE, differentially expressed; miRNA, microRNA; up, upregulated; down, downregulated. 


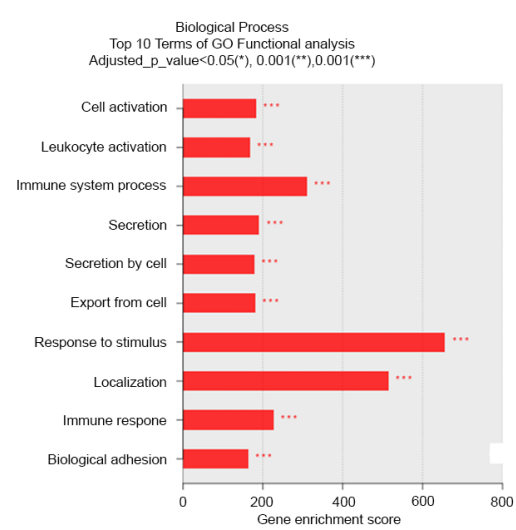

B

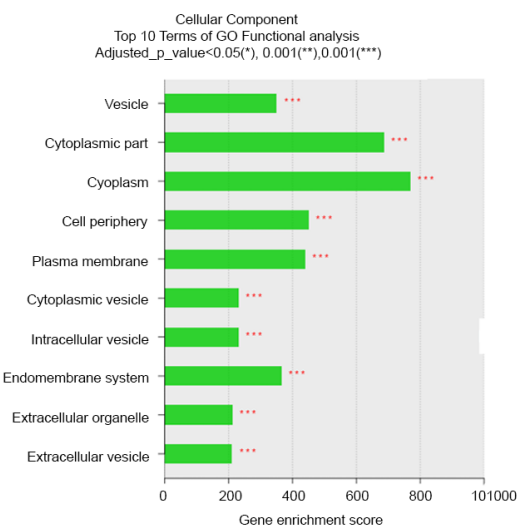

C

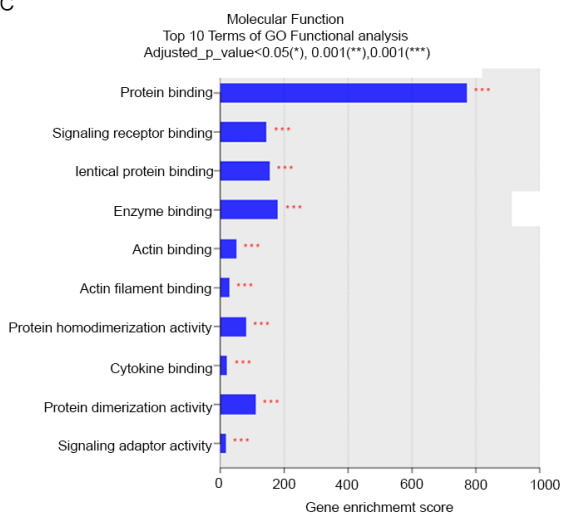

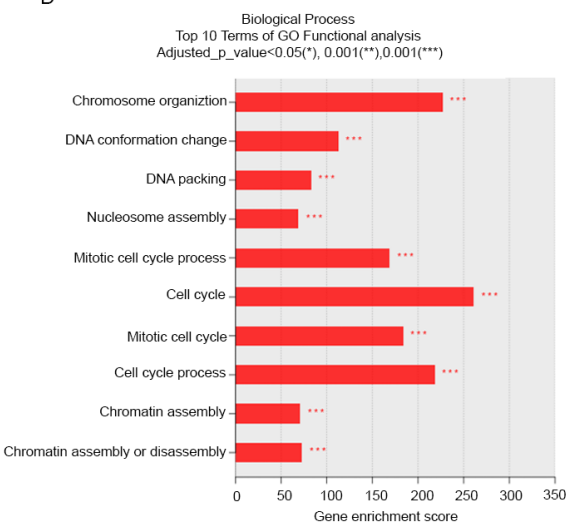

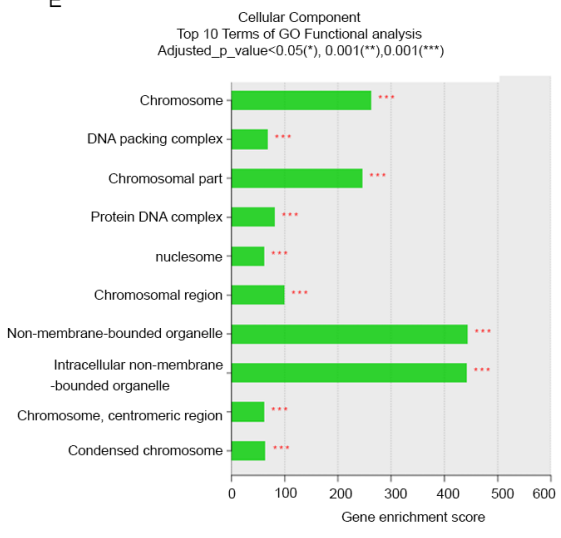

$\mathrm{F}$

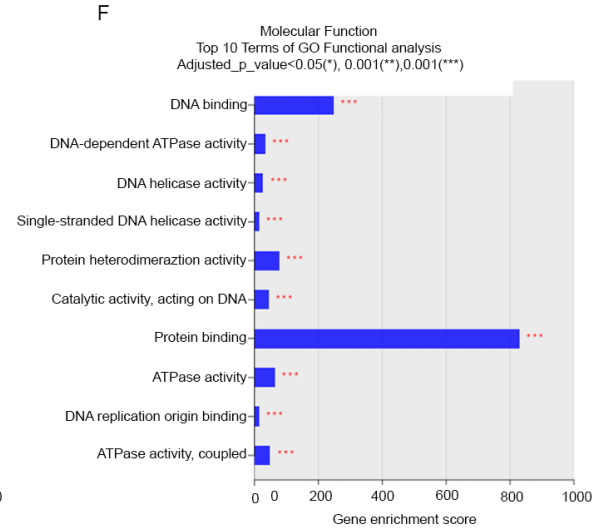

G

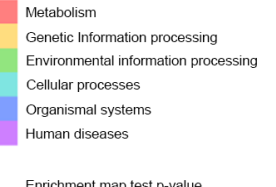

$\begin{array}{lll}\text { Enrichment map test } p-\text { value } \\ P<=0.001 & P<=0.01 \quad P<=0.05 & P>0.05\end{array}$



Figure 3. GO enrichment and KEGG pathway analysis based on differentially expressed genes in the proliferative and secretory endometria. Top 10 upregulated GO terms enriched for (A) biological processes, (B) cellular components and (C) molecular functions. Top 10 downregulated GO terms enriched for (D) biological processes, (E) cellular components and (F) molecular functions. (G) The top 20 KEGG pathways based on the up- and downregulated genes. GO, Gene Ontology; KEGG, Kyoto Encyclopedia of Genes and Genomes.

RNA 1 (HAND2-AS1) were upregulated in the mid-secretory endometrium, whereas DLX6 antisense RNA1 (DLX6-AS1), TCF21 antisense RNA inducing promoter demethylation (TARID), and HHIP antisense RNA1 (HHIP-AS1) were downregulated (Fig. 4A). In addition, hsa-miR-29b, hsa-miR-30b, and hsa-miR-30d were upregulated in themid-secretory endometrium, whereas $h s a-m i R-182$ and $h s a-m i R-183$ were downregulated (Fig. 4B). Of the DE mRNAs, ribosomal modification protein RimK like family member B (RIMKLB), cysteine-rich secretory protein 3 (CRISP3), and thioredoxin interacting protein (TXNIP) were significantly upregulated in the mid-secretory endometrium, whereas neuronal regeneration-related protein (NREP) and progesterone receptor membrane component 1 (PGRMC1) were downregulated (Fig. 4C).
In silico identification of $m R N A s$ associated with endometrial receptivity. To investigate the mRNAs associated with endometrial receptivity, we performed in silico analysis and compared the DE mRNAs that we identified with those reported by three previous studies based on RNA-seq (10-12). Of the 2,154 DE mRNAs we identified, 501 were identified in at least two previous studies; 1,121 were uniquely identified in this study, of which 565 were upregulated and 556 were downregulated. Moreover, 98 DE mRNAs, of which 60 were upregulated and 38 were downregulated in the mid-secretory endometrium, were commonly identified in all four studies (Fig. 5A; Table SVI). Furthermore, KEGG pathway enrichment analysis revealed that these 98 genes were significantly involved in tryptophan metabolism, metabolic pathways, 
A
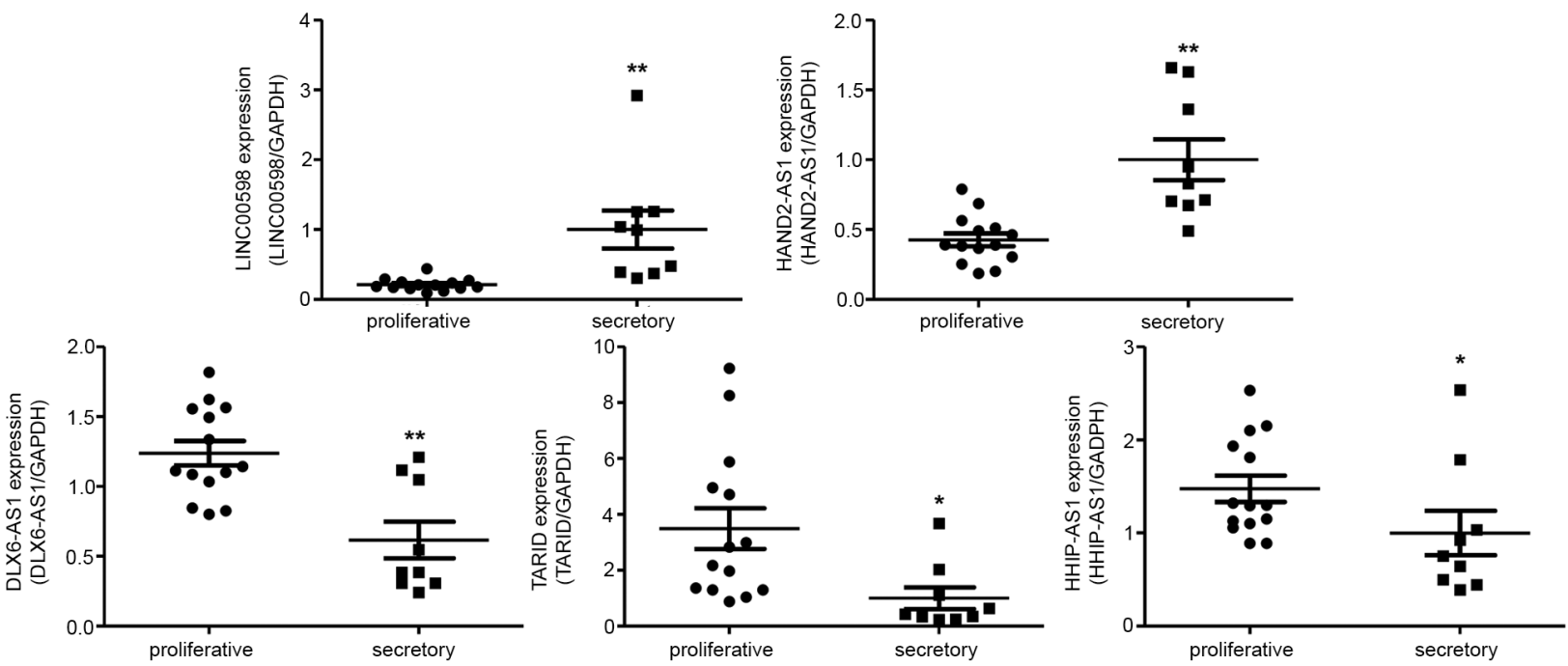

B
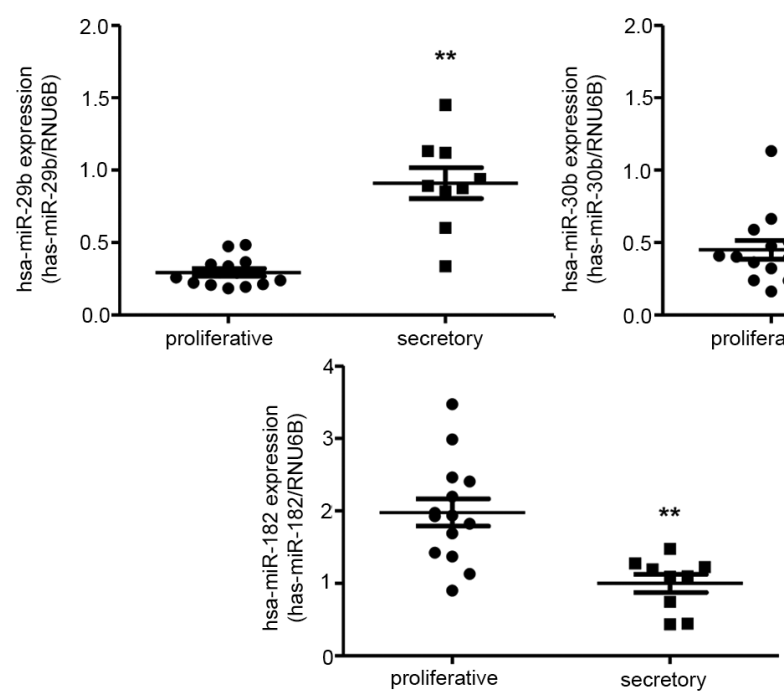

C

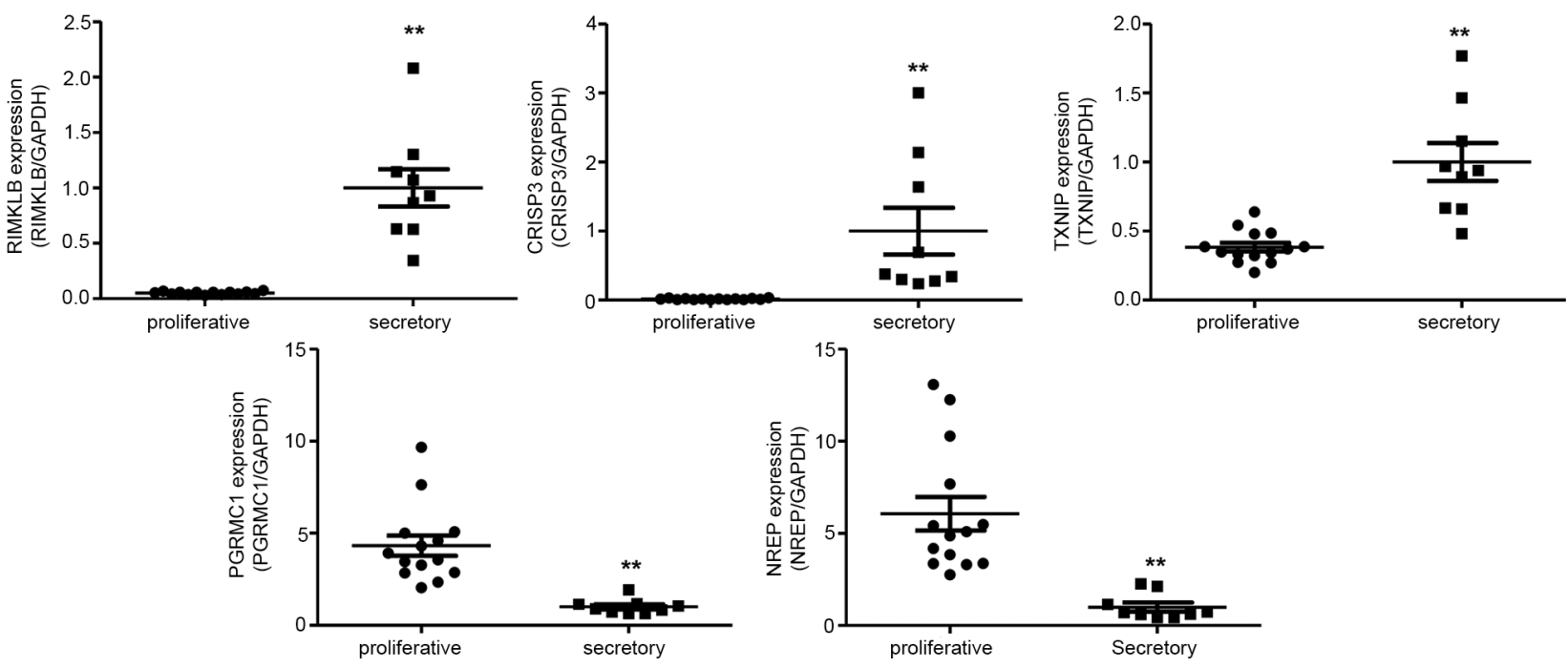

Figure 4. Quantitative PCR validation of DE lncRNAs, miRNAs and mRNAs in proliferative and mid-secretory endometria. The expression of (A) five IncRNAs, (B) five miRNAs and (C) five mRNAs were detected by quantitative PCR in proliferative and mid-secretory endometrium samples. Data are presented as the normalized fold change. Experiments were repeated in triplicate, and values are expressed as mean \pm SEM. " $\mathrm{P}<0.05$ and ${ }^{* *} \mathrm{P}<0.01$ vs. proliferative. IncRNA, long non-coding RNA; miRNA or miR, microRNA.

and FoxO signaling (Fig. 5B). In the category of tryptophan metabolism, $A O X 1, M A O A$, and IDOI expression levels were highly increased in the mid-secretory endometrium compared with those in the proliferative endometrium, whereas in the category of metabolic pathways, AOX1, MAOA, RIMKLB, IDO1,NNMT, ARG2, HAL, GALNT13, PLA2G16, and ADCY1 
A

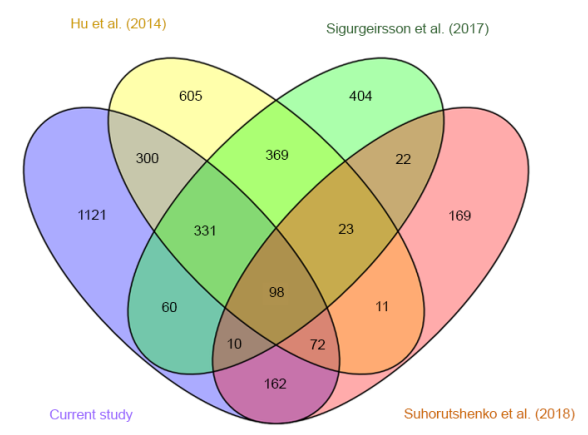

C

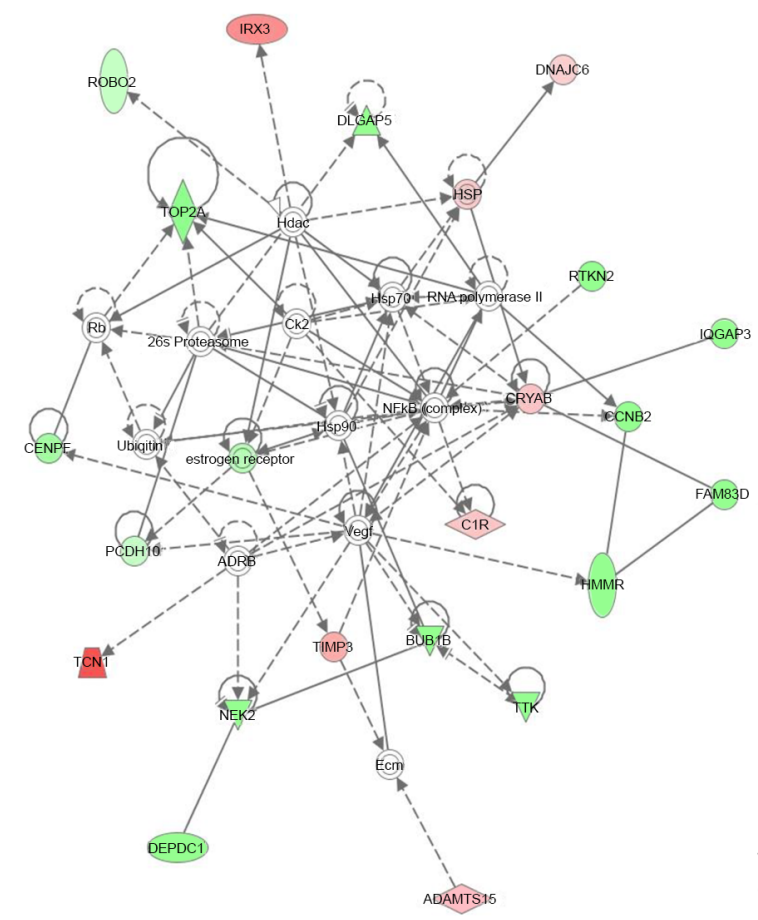

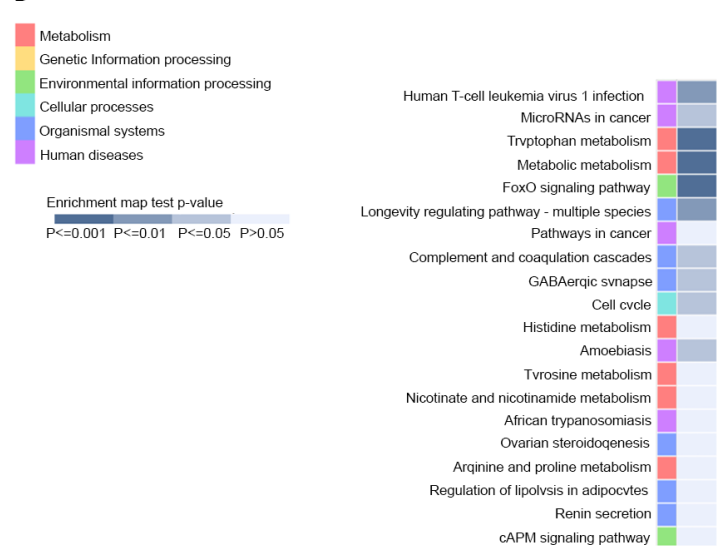

D

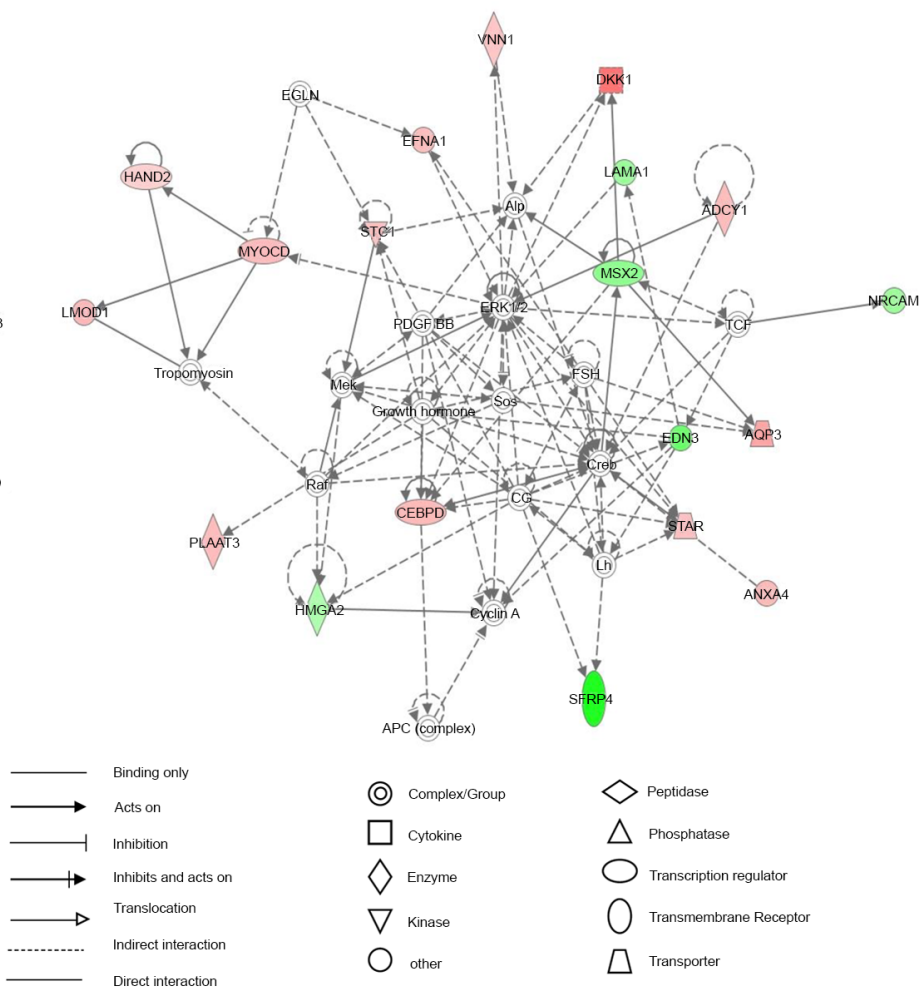

Figure 5. Transcriptome and bioinformatics analysis of DEGs from the current study and previous RNA-sequencing results. (A) Venn diagram of the number of DEGs in the four assessed studies. (B) Top 20 KEGG pathways for the 98 common genes of the four studies. Ingenuity pathway analysis network generated from 98 common genes from the four studies. (C) Network related with 'cell cycle, cellular assembly and organization, DNA replication, and recombination and repair'. (D) Network related with 'cell death and survival, cellular development, and connective tissue development and function'. DEGs, differentially expressed genes.

were upregulated and GALNT12 was downregulated in the mid-secretory endometrium. Conversely, in FoxO signaling, SOD2, FBXO32, GABARAPL1, and BCL6 expression increased in the mid-secretory endometrium, whereas $C C N B 2$ expression decreased.

To investigate changes in biological pathways based on these 98 common DE mRNAs, we performed IPA and identified five networks, the first of which had a score of 44 and included 23 focus genes (Fig. 5C) whose main functions were in 'cell cycle,' 'cellular assembly and organization,' 'DNA replication,' and 'recombination and repair.' In this network, ADAMTS15, C1R, CRYAB, DNAJC6, HSP, IRX3, TCN1, and TIMP3 mRNAs were upregulated in the mid-secretory endometrium, whereas $B U B 1 B, C C N B 2, C E N P F, D E P D C 1$, $D L G A P 5$, estrogen receptor, FAM83D, HMMR, IQGAP3, NEK2, PCDH10, RTKN2, ROBO1, TOP2A, and TTK mRNAs were downregulated. The second network had a score of 39 and included 19 focus genes (Fig. 5D), whose main functions were in 'cell death and survival,' 'cellular development,' and 'connective tissue development and function.' In this network, ADCY1, ANXA4, AQP3, CEBPD, DKK1, EFNA1, HAND2, LOMD1, MYOCD, PLAAT3, STAR, STC1, and VNN1 mRNAs were upregulated in the mid-secretory endometrium, whereas EDN3, HMGA2, LAMA1, MSX2, NRCAM, and SFRP4 mRNAs were downregulated. These results suggest that the above networks were closely related to endometrial receptivity. 
A

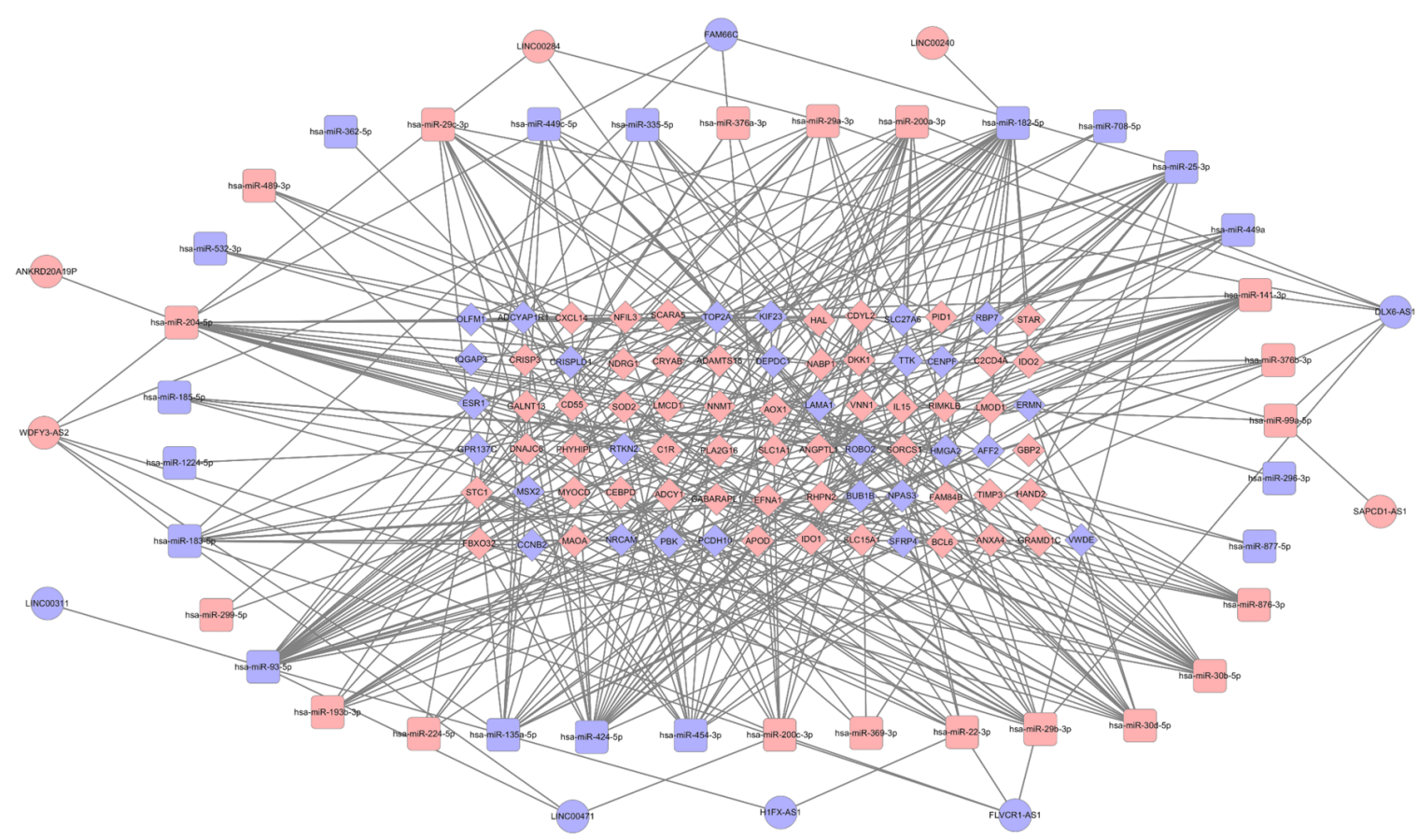

B



C



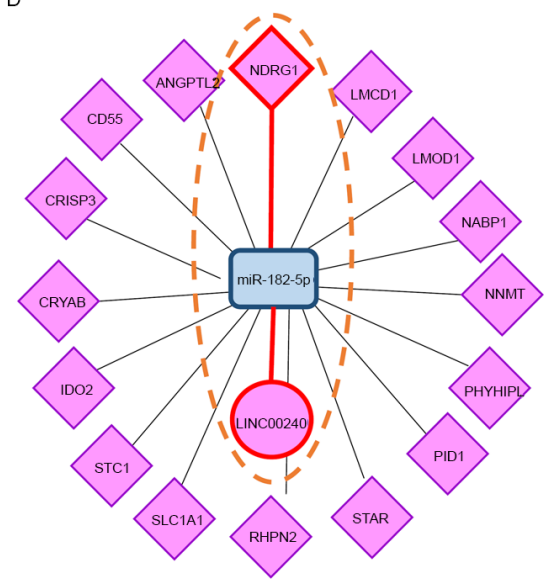

Figure 6. ceRNA network related to endometrial receptivity. (A) lncRNA/miRNA/mRNA ceRNA network for proliferative and mid-secretory endometria. (B) ceRNA subnetwork for DLX6-AS1. (C) ceRNA subnetwork for WDFY3-AS2. (D) ceRNA subnetwork for LINC00240. Circles, squares and diamonds represent lncRNAs, miRNAs and genes, respectively. Upregulated genes are indicated in red and downregulated genes are indicated in blue. Dotted lines emphasize important networks related to endometrial receptivity. IncRNA, long non-coding RNA; miRNA, microRNA; ceRNA, competitive endogenous RNA.

ceRNA network construction. To better understand the pivotal combined roles of DE IncRNAs, miRNAs, and mRNAs, we constructed and visualized a ceRNA network (Fig. 6A). Eleven DE IncRNAs (five upregulated and six downregulated) interacted with 13 DE miRNAs from the miRcode database, and 36 DE miRNAs (19 upregulated and 17 downregulated) interacted with $79 \mathrm{DE}$ mRNAs (51 upregulated and 28 downregulated) retrieved from the mirDIP database. These 11 DE lncRNAs, 36 DE miRNAs, and 79 DE mRNAs were then used to establish a ceRNA network that consisted of 126 nodes and 370 edges, where the number of edges derived from nodes indicates regulatory interactions between RNAs and the importance of biological functions. The top hub
RNAs included two lncRNAs ( $W D F Y 3-A S 2$, upregulated; DLX6-AS1, downregulated) and six miRNAs (hsa-miR-141, hsa-miR-200a, and hsa-miR-204: Upregulated; $h s a-m i R-93$, hsa-miR-182, and hsa-miR-424: Downregulated). Of the hub lncRNAs, DLX6-AS1 formed a connecting network with two DE miRNAs and 10 DE mRNAs (Fig. 6B). WDFY3-AS2 was also associated with five DE miRNAs and 11 DE mRNAs (Fig. 6C). Finally, LINC00240 was associated with one DE miRNA and 16 DE mRNAs (Fig. 6D).

We also identified hub RNAs forming three axes: The DLX6-AS1/miR-141 or miR-200a/OLFM1 axis, WDFY3-AS2/miR-135a or miR-183/STC1 axis, and LINC00240/miR-182/NDRG1 axis, which may be related to 
A
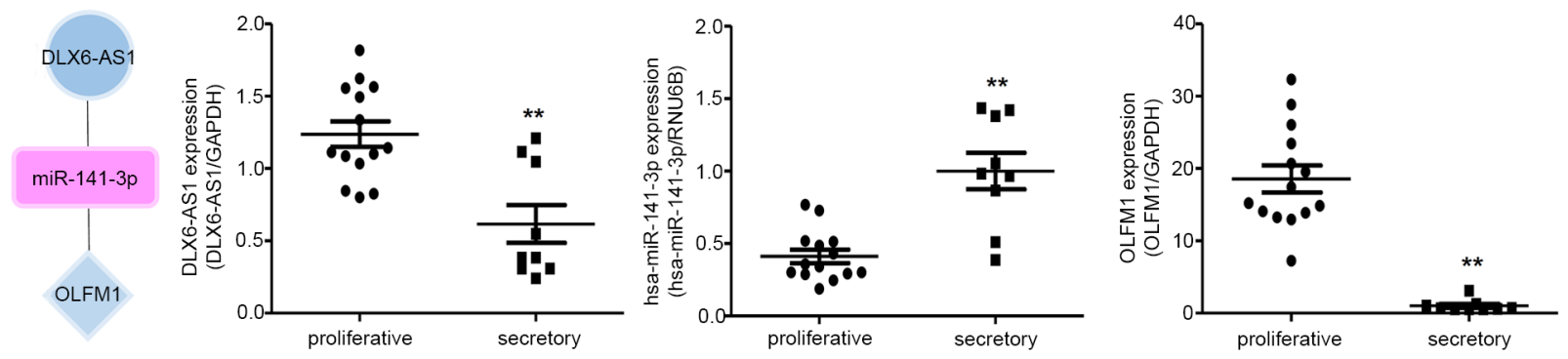

B
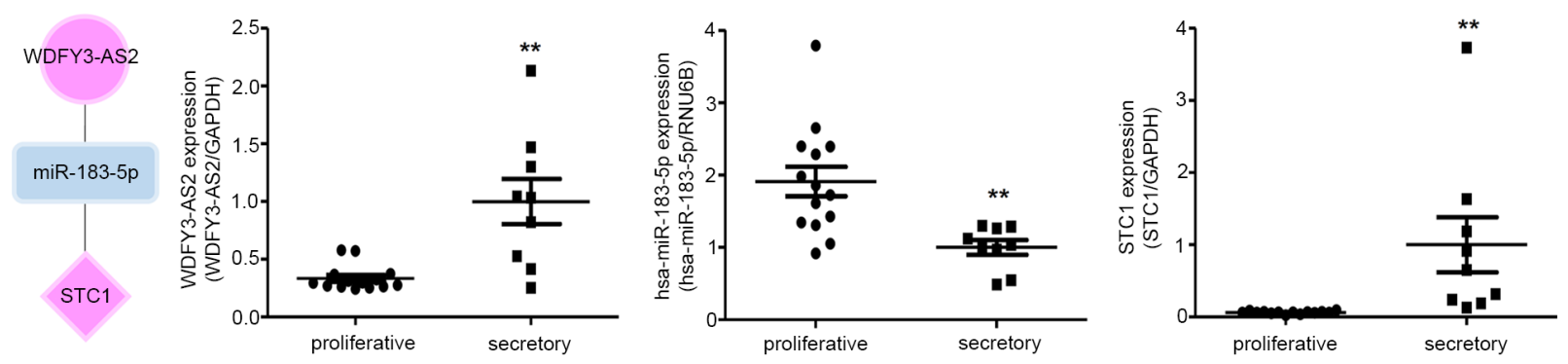

C
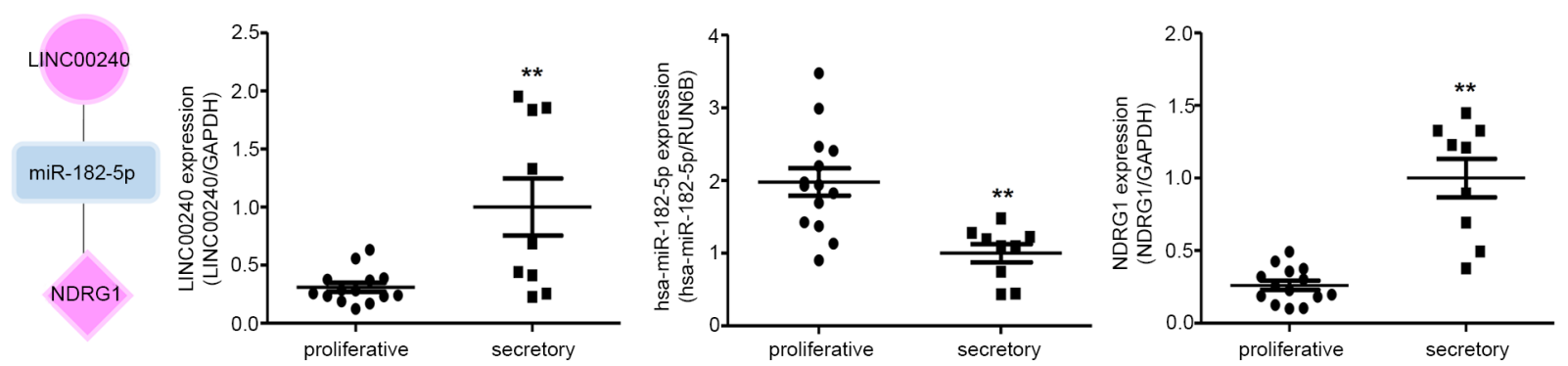

Figure 7. RNA expression correlation between the three ceRNA subnetworks in proliferative and secretory endometria. The expression of (A) DLX6-AS1, hsa-miR-141-3p and OLFM; (B) WDFY3-AS2, hsa-miR-183-5p and STC1; and (C) LINC00240, hsa-miR-182-5p and NDRG1 (C) was detected by quantitative PCR in proliferative and secretory endometria. The circles, squares and diamonds represent lncRNAs, miRNAs and genes, respectively. Upregulated genes are indicated in red and downregulated genes are indicated in blue. All data are presented as the mean $\pm \mathrm{SEM}$, and experiments were repeated in triplicate. ${ }^{* *} \mathrm{P}<0.01$. ceRNA, competitive endogenous RNA; IncRNA, long non-coding RNA; miRNA, microRNA $>=$.

endometrial receptivity (Fig. 6B, C and D; dotted lines). To evaluate the RNA expression correlation between the tree axes, we examined the relative RNA expression by RT-qPCR in proliferative and mid-secretory phase endometrial tissues. As shown in Fig. 7, the miRNA expression patterns were negatively correlated with IncRNAs in both proliferative and secretory tissues. The mRNA expression patterns were contrary to those of miRNA. In addition, three hub RNAs showed different expression patterns between secretory and proliferative tissues. Taken together, these results indicate that the ceRNA network might play crucial roles in the regulation of endometrial receptivity.

\section{Discussion}

Many studies have investigated the gene expression profiles of the prereceptive and receptive endometria to identify the transcriptome related to endometrial receptivity; however, the interactions between genes and ncRNAs associated with endometrial receptivity have remained unclear. In this study, we investigated whether the interplay among genes, miRNAs, and IncRNAs affect molecular signaling associated with endometrial receptivity. Of the top 10 up- and downregulated DE mRNAs identified in our study (Table II), eight upregulated genes and three downregulated genes belonged to 57 genes that were previously proposed as biomarkers of human endometrial receptivity (30). Of the DEGs, CXC motif chemokine ligand (CXCL14), transcobalamin 1 (TCN1), complement component 4 binding protein alpha (C4BPA), aldehyde oxidase 1 (AOXI), Dickkopf Wnt signaling pathway inhibitor $1(D K K 1)$, secreted frizzled-related protein (SFRP4), endothelin 3 (EDN3), and olfactomedin 1 (OLFM1) were related to 238 endometrial receptivity analysis array genes (31). The PAEP gene showed the greatest upregulation in the mid-secretory endometrium in this study. This gene has been reported to display negative or low expression in the proliferative endometrium (32) and may serve as a candidate biomarker for endometrial receptivity $(33,34)$. SFRP4 modulates Wnt signaling and is more highly expressed in proliferative endometrium compared with that in secretory endometrium (35); upregulation of SFRP4 in the placenta is associated with severe preeclampsia (36). OLFM1 is an extracellular matrix protein that is expressed at significantly lower levels in the secretory endometrium than in the proliferative endometrium and negatively regulates 
Table IV. Top 10 differentially expressed upregulated and downregulated microRNAs with the highest fold change.

A, Upregulated microRNAs

\begin{tabular}{lcl}
\hline ID & FC & P-value \\
\hline hsa-miR-4525 & 11.6143 & 0.0054 \\
hsa-miR-489-3p & 7.7917 & 0.0392 \\
hsa-miR-1247-5p & 5.7667 & $2.39 \times 10^{-5}$ \\
hsa-miR-193b-3p & 5.2862 & 0.0098 \\
hsa-miR-941 & 4.9600 & 0.0088 \\
hsa-miR-876-3p & 4.2922 & 0.0103 \\
hsa-miR-224-5p & 4.1808 & $5.31 \times 10^{-10}$ \\
hsa-miR-376b-3p & 4.0789 & 0.0491 \\
hsa-miR-30d-3p & 4.0318 & 0.0044 \\
hsa-miR-642a-3p & 3.9458 & 0.0484 \\
\hline
\end{tabular}

B, Downregulated microRNAs

\begin{tabular}{lcc}
\hline ID & FC & P-value \\
\hline hsa-miR-149-3p & -8.6235 & 0.0477 \\
hsa-miR-362-5p & -7.2379 & 0.0117 \\
hsa-miR-335-3p & -5.5844 & 0.0469 \\
hsa-miR-486-3p & -5.2467 & 0.0075 \\
hsa-miR-95-3p & -5.2448 & 0.0140 \\
hsa-miR-365b-5p & -5.0526 & 0.0041 \\
hsa-miR-3179 & -4.5559 & 0.0242 \\
hsa-miR-296-3p & -4.2086 & 0.0280 \\
hsa-miR-135a-5p & -4.1637 & 0.0404 \\
hsa-miR-1224-5p & -4.1609 & 0.0372 \\
\hline
\end{tabular}

FC, fold change; miR, microRNA.

spheroid attachment between choriocarcinoma JAr cells and Ishikawa cells (37). Moreover, human chorionic gonadotropin secreted by pre-implantation embryos significantly downregulates OLFM1 expression (38). Thus, the genes identified in this study may be closely related to endometrial receptivity.

Of the 247 DE IncRNAs identified in this study, RIMKLB transcript variant 3 showed the highest upregulation, whereas MMP11 transcript variant 2 showed the greatest downregulation. Among the top 10 up- and downregulated lncRNAs (Table III), LINC01320 was upregulated in the endometrium during the WOI, consistent with findings of previous studies (11,12). KCNIP4-IT1 and DLX6-AS1 are also downregulated in the secretory endometrium (11), whereas DLX6-ASI is overexpressed in the placenta of patients with preeclampsia and is associated with reduced trophoblast proliferation, migration, and invasion (39). However, the correlations between lncRNAs and implantation are not clear. Of the 67 DE miRNAs identified in our study, $h s a-m i R-4525$ showed the highest upregulation, and $h s a-m i R-149-3 p$ showed the most downregulation. Mmu-miR-193 influences embryo implantation by modulating growth factor receptor-bound protein 7 expression in mice (40). Similarly, miR-30d knockout reduces the implantation rate in mice (41), and $m i R-135 a$ and $m i R-135 b$ are downregulated in receptive endometrium (11). FOXO1 is a decidualization marker that is regulated post-transcriptionally by $m i R-135 a$ in human melanoma cells (42); however, the roles of miRNAs in the endometrium remain unclear.

Of top 20 enriched KEGG pathways, adhesion molecules are known to be expressed in human endometrial epithelial cells and can be used as markers of endometrial receptivity (43). Focal adhesion, cAMP signaling, and RAP1 signaling pathways were significantly enhanced in the mid-secretory endometrium. Consistent with this, Kusama et al demonstrated that RAP1 is crucial for cAMP-mediated decidualization in rat and human endometrial stromal cells (44). Thus, focal adhesion, cAMP signaling, and RAP1 signaling may be important for decidualization during the receptive phase.

Of the 2,154 DEGs identified herein, 1,121 mRNAs were uniquely identified in this study. Recently, 18,210 structural variations have been identified in the Korean human genome compared with the human reference genome GRCh38, and most of the insertions among structural variants are causes of the variance in the transcriptome (45). Therefore, as compared with previously published data, the mRNAs that were uniquely expressed in our study were probably derived from differences between human populations. Moreover, the study endometrium donors (about 37.5 years of age) were about 10 years older than donors in previous studies (about 28 years of age). Aging regulates complex biological processes in humans (46). Therefore, differences in the age of volunteers may have contributed to the uniquely expressed mRNA. By analyzing previously published RNA-seq expression profiling studies, we identified 98 common genes involved in mid-secretory endometrial function, of which 34 were shared with the endometrial receptivity analysis array, a commercial tool used for endometrial receptivity diagnosis (31). Moreover, 50 of these 98 common genes were identical to 57 genes proposed as putative receptivity markers through meta-analysis by Altmäe et al (30). Therefore, we propose that the 98 common genes identified in this study may be related to endometrial receptivity. In the KEGG pathways of these 98 common genes, previous studies have reported the expression patterns of AOX1, MAOA, IDO1, NNMT, ARG2, HAL, GALNT12, SOD2, GABARAPL1, BCL6, and CCNB2 in the endometrium (30,31). In particular, the localization and involvement of AOX1, MAOA, IDO1, ARG2, NNMT, and BCL6 in the mid-secretory endometrium have been well described (30). MAOA is known to affect human endometrial receptivity, and its expression may be altered by inadequate decidualization (47); indeed, patients who experience implantation failure display decreased MAOA expression $(48,49)$. IDO1 inhibits the expression of the decidualization marker genes $\mathrm{Prl}$ and $\mathrm{Igfbpl}$ in mice under in vitro decidualization (50). FOXO1 is an important cAMP-dependent transcription factor in decidualizing human endometrial stromal cells and exerts antioxidant properties by targeting and regulating SOD2 expression (51-55). SOD2 and FoxO1 expression is induced during the differentiation of the stromal compartment in the mid- to late-secretory phase of the cycle and these proteins are expressed in decidualizing endometrial stromal cells in culture (52). In addition, decidualization is associated with the induction of various free radical scavengers, including SOD2 (56). Although MAOA, IDO1, 
and SOD2 are known to participate in decidualization and endometrial receptivity, further studies are required to determine the effects of the other genes on endometrial receptivity.

In this study, we also demonstrated that networks related to the cell cycle, DNA replication, DNA recombination, and DNA repair for cell proliferation were mostly downregulated in the mid-secretory endometrium, whereas networks involved in cell survival, cellular development, and connective tissue development were upregulated. Within these networks, the DKK1 protein has been shown to affect spheroid attachment on endometrial epithelial cells, and MSX2 knockout directly affects endometrial receptivity and embryo implantation in mice $(57,58)$. Therefore, these networks and the genes involved in them may play important roles in endometrial receptivity and implantation.

LncRNA/miRNA/mRNA interactions are known to form a network of ceRNAs with key roles in biological networks. Until recently, most studies of ceRNAs have been related to cancer, including tumor diagnosis, prognosis, and targeted treatments, with few studies examining endometrial receptivity by constructing ceRNA networks. Recently, in patients with and without endometriosis, Wang and Yu identified four ceRNA networks as biomarkers for endometrial receptivity (59). Similarly, Xu et al identified potential novel biomarkers for repeated implantation failure from a ceRNA network constructed from DE RNAs (60). In this study, we successfully constructed ceRNA networks to identify the RNA interactions that affect endometrial receptivity and discovered that the top hub RNAs includ two IncRNAs and six miRNAs. Of the hub lncRNAs, DLX6-ASI acts as a sponge for many miRNAs and is significantly overexpressed in various cancers, including cervical cancer $(61,62)$. DLX6-AS1 is also upregulated in the placenta of patients with preeclampsia and negatively regulates the proliferation, migration, and invasion of trophoblasts (39). Of the hub miRNAs, $m i R-141-3 p$ can directly sponge DLX6-ASI (63), and miR-141 is upregulated in endometriomas compared with the eutopic endometrium of patients with endometriosis (64). Interestingly, $m m u$-miR-141 affects the proliferation of endometrial cells and the number of embryo implantation sites in mice, suggesting essential roles in embryo implantation (65). $m i R-141$ belongs to the $m i R-200$ family, which also includes miR-200a, miR-200b, miR-200c, and $\mathrm{miR}-429 \mathrm{miR}-200 \mathrm{a} / \mathrm{b} / \mathrm{c}$ are upregulated during endometrial stromal cell decidualization in vitro (66), whereas OLFM1 has been reported as a target of $m i R-141$ and $m i R-200 a$ in human gastric cancer cells (67). In a trophoblastic spheroid (JAr)-endometrial epithelial cell (Ishikawa) co-culture model, recombinant OLFM1 protein treatment suppressed the attachment of JAr spheroids onto the Ishikawa cell monolayer, suggesting that OLFM1 inhibits endometrial receptivity (68). Therefore, the DLX6-AS1/miR-141 or miR-200a/OLFM1 axes may be important regulators of endometrial receptivity.

Endometrial cell proliferation decreased between the proliferative to mid-secretory phases; however, uncontrolled endometrial epithelial cell proliferation can lead to implantation failure and has been observed in the eutopic secretory endometrium of patients with endometriosis (69). Therefore, endometrial cell proliferation is closely related to pregnancy. WDFY3-AS2, which was identified from our ceRNA network, has no known endometrial function but has been reported to be related to cancer progression. For example, WDFY3-AS2 inhibits cancer cell proliferation and invasion $(70,71)$, and $m i R-135 a$, which is a potential target of WDFY3-AS in diffuse glioma (72), promotes cancer cell proliferation, migration, and invasion (73) and directly suppresses FOXO1 in hepatocellular carcinoma cells (74). Other pregnancy-related studies showed strong expression of stanniocalcin (STC) 1 and STC2 mRNA in decidualized rat cells, suggesting that STC1 and STC2 play important roles in implantation and decidualization (75). In humans, STC1 is upregulated in the secretory endometrium compared with that in the proliferative endometrium, and is dysregulated in the eutopic endometrium of patients with endometriosis, suggesting its roles in the pathogenesis of decidualization defects (76). Therefore, accumulating evidence indicates that the WDFY3-AS2/miR-135a/STC1 axis may be involved in endometrial stromal cell decidualization. The ceRNA network in this study revealed a potential, previously unknown interaction between WDFY3-AS2 and miR-183, which is decreased in vitro during human endometrial stromal cell decidualization (77). In addition, miR-183 suppresses FOXO1 in non-small cell lung cancer (NSCLC), significantly increasing NSCLC growth in vitro and in vivo (78). Recently, Akbar et al suggested that miR-183-5p may be a potential biomarker for endometrial receptivity (79), and STC1 has been reported as a potential target of miR-183-5p in bladder cancer (80). Thus, our study suggests that the WDFY3-AS2/miR-183-5p/STC1 axis could be a biomarker for endometrial receptivity.

Although LINC00240 was more highly expressed in the secretory endometrium than in the proliferative endometrium in this study, no interaction between LINC00240 and miR-182 has been reported. LINC00240 promotes cell proliferation, invasion, and migration in gastric and cervical cancer $(81,82)$, and miR-182 suppresses FOXO1 expression in endometrial cancer cells (83). In addition, $\mathrm{N}$-myc downregulated gene 1 (NDRG1) directly affects pregnancy in mice, and reduced NDRG1 expression has been reported in decidual samples from patients with recurrent miscarriage (84). Thus, the LINC00240/miR-182/NDRG1 axis may also play important roles in endometrial receptivity.

There were some limitations to this study. First, the sample size was small, and tissue heterogeneity could have limited the generalization of the results. Additionally, sample collection was based on menstrual cycle history, ultrasound finding, and histological confirmation rather than serum luteinizing hormone concentrations, which are generally used for cycle dating. In fact, endometrial receptivity is the receptive status of the endometrium that allows the embryo to implant and is usually compared between endometria of fertile and infertile patients in the WOI. Therefore, we are currently collecting endometrium samples from infertile patients to further explore genes associated with endometrial receptivity.

In conclusion, the ceRNA networks constructed in this study may partially explain the regulatory mechanisms underlying endometrial receptivity; however, further studies are required to define the relationships between these ceRNA networks and endometrial receptivity.

\section{Acknowledgements}

Not applicable. 


\section{Funding}

The present study was supported by the Basic Science Research Program through the National Research Foundation of the Ministry of Education, Korea (grant no. NRF-2017R1A6A1A03015713).

\section{Availability of data and materials}

The datasets used an/or analyzed are available from the corresponding author on reasonable request. The smRNA sequencing data that support the findings of this study are openly available in the GEO database (https://www.ncbi.nlm. nih.gov/geo/query/acc.cgi?acc=GSE167325).

\section{Authors' contributions}

JK and SRP conceived the current study and designed the experiments. THK provided resources. SLY, THK and YHH designed the methods. SLY, YHH, YK and DUJ constructed the figures. DCL performed ingenuity pathway analysis. SLY, YHH, YK and DUJ validated the genes, lncRNAs and miRNAs identified from RNA-seq. SLY, THK, YHH and SRP wrote the original draft of the manuscript. SLY, THK, JK and SRP wrote and reviewed the manuscript. JK and SRP supervised the current study. JK and SRP acquired funding. All authors have read and approved the final manuscript, and agree to the published version of the manuscript. JK and SRP confirm the authenticity of all the raw data.

\section{Ethics approval and consent to participate}

The current study was approved by the Bioethics Committee of KYU (Institutional Review Board File No. 2018-11-007-005). All patients provided written informed consent.

\section{Patient consent for publication}

Not applicable.

\section{Competing interests}

The authors declare that they have no competing interests.

\section{References}

1. Psychoyos A: Hormonal control of ovoimplantation. Vitam Horm 31: 201-256, 1973.

2. Simon C, Martin JC and Pellicer A: Paracrine regulators of implantation. Best Pract Res Clin Obstet Gynaecol 14: 815-826, 2000.

3. Aplin JD: The cell biological basis of human implantation. Best Pract Res Clin Obstet Gynaecol 14: 757-764, 2000.

4. Cha J, Sun X and Dey SK: Mechanisms of implantation: Strategies for successful pregnancy. Nat Med 18: 1754-1767, 2012.

5. Carson DD, Lagow E, Thathiah A, Al-Shami R, Farach-Carson MC, Vernon M, Yuan L, Fritz MA and Lessey B: Changes in gene expression during the early to mid-luteal (receptive phase) transition in human endometrium detected by high-density microarray screening. Mol Hum Reprod 8: 871-879, 2002.

6. Kao LC, Tulac S, Lobo S, Imani B, Yang JP, Germeyer A, Osteen K, Taylor RN, Lessey BA and Guidice LC: Global gene profiling in human endometrium during the window of implantation. Endocrinology 143: 2119-2138, 2002.
7. Borthwick JM, Charnock-Jones DS, Tom BD, Hull ML, Teirney R, Phillips SC and Smith SK: Determination of the transcript profile of human endometrium. Mol Hum Reprod 9: 19-33, 2003.

8. Riesewijk A, Martin J, van Os R, Horcajadas JA, Polman J, Pellicer A, Mosselman S and Simón C: Gene expression profiling of human endometrial receptivity on days LH+2 versus LH+7 by microarray technology. Mol Hum Reprod 9: 253-264, 2003.

9. Mirkin S, Arslan M, Churikov D, Corica A, Diaz JI, Williams S, Bocca $S$ and Oehninger S: In search of candidate genes critically expressed in the human endometrium during the window of implantation. Hum Reprod 20: 2104-2117, 2005.

10. Hu S, Yao G, Wang Y, Xu H, Ji X, He Y, Zhu Q, Chen Z and Sun Y: Transcriptomic changes during the pre-receptive to receptive transition in human endometrium detected by RNA-Seq. J Clin Endocrinol Metab 99: E2744-E2753, 2014.

11. Sigurgeirsson B, Amark H, Jemt A, Ujvari D, Westgren M, Lundeberg $\mathrm{J}$ and Gidlöf S: Comprehensive RNA sequencing of healthy human endometrium at two time points of the menstrual cycle. Biol Reprod 96: 24-33, 2017.

12. Suhorutshenko M, Kukushkina V, Velthut-Meikas A, Altmäe S, Peters M, Mägi R, Krjutškov K, Koel M, Codoñer FM, Martinez-Blanch JF, et al: Endometrial receptivity revisited: Endometrial transcriptome adjusted for tissue cellular heterogeneity. Hum Reprod 33: 2074-2086, 2018.

13. Gomez E, Ruiz-Alonso M, Miravet J and Simon C: Human endometrial transcriptomics: Implications for embryonic implantation. Cold Spring Harb Perspect Med 5: a022996, 2015.

14. Yamamura S, Imai-Sumida $\mathrm{M}$, Tanaka $\mathrm{Y}$ and Dahiya R: Interaction and cross-talk between non-coding RNAs. Cell Mol Life Sci 75: 467-484, 2018

15. Guttman M, Amit I, Garber M, French C, Lin MF, Feldser D, Huarte M, Zuk O, Carey BW, Cassady JP, et al: Chromatin signature reveals over a thousand highly conserved large non-coding RNAs in mammals. Nature 458: 223-227, 2009.

16. Fan LJ, Han HJ, Guan J, Zhang XW, Cui QH, Shen H and Shi C: Aberrantly expressed long noncoding RNAs in recurrent implantation failure: A microarray related study. Syst Biol Reprod Med 63: 269-278, 2017.

17. Feng C, Shen JM, Lv PP, Jin M, Wang LQ, Rao JP and Feng L: Construction of implantation failure related lncRNA-mRNA network and identification of IncRNA biomarkers for predicting endometrial receptivity. Int J Biol Sci 14: 1361-1377, 2018.

18. Chen MY, Liao GD, Zhou B, Kang LN, He YM and Li SW: Genome-wide profiling of long noncoding RNA expression patterns in women with repeated implantation failure by RNA sequencing. Reprod Sci 26: 18-25, 2019.

19. Lim LP, Lau NC, Garrett-Engele P, Grimson A, Schelter JM, Castle J, Bartel DP, Linsley PS and Johnson JM: Microarray analysis shows that some microRNAs downregulate large numbers of target mRNAs. Nature 433: 769-773, 2005.

20. Friedman RC, Farh KK, Burge CB and Bartel DP: Most mammalian mRNAs are conserved targets of microRNAs. Genome Res 19: 92-105, 2009.

21. Rižner TL: Discovery of biomarkers for endometrial cancer: Current status and prospects. Expert Rev Mol Diagn 16: 1315-1336, 2016.

22. Wilczynski M, Danielska J, Dzieniecka M, Szymanska B, Wojciechowski M and Malinowski A: Prognostic and clinical significance of miRNA-205 in endometrioid endometrial cancer. PLoS One 11: e0164687, 2016.

23. Bashti O, Noruzinia M, Garshasbi M and Abtahi M: miR-31 and miR-145 as potential non-invasive regulatory biomarkers in patients with endometriosis. Cell J 20: 293, 2018.

24. Salmena L, Poliseno L, Tay Y, Kats L and Pandolfi PP: A ceRNA hypothesis: The Rosetta Stone of a hidden RNA language? Cell 146: 353-358, 2011

25. Noyes RW, Hertig AT and Rock J: Reprint of: Dating the endometrial biopsy. Fertil Steril 112 (Suppl 1): e93-e115, 2019.

26. Pertea M, Kim D, Pertea GM, Leek JT and Salzberg SL: Transcript-level expression analysis of RNA-seq experiments with HISAT, StringTie and Ballgown. Nat Protoc 11: 1650-1667, 2016.

27. Love MI, Huber W and Anders S: Moderated estimation of fold change and dispersion for RNA-seq data with DESeq2. Genome Biol 15: 550, 2014.

28. Shannon P, Markiel A, Ozier O, Baliga NS, Wang JT, Ramage D, Amin N, Schwikowski B and Ideker T: Cytoscape: A software environment for integrated models of biomolecular interaction networks. Genome Re 13: 2498-2504, 2003. 
29. Achache $\mathrm{H}$ and Revel A: Endometrial receptivity markers, the journey to successful embryo implantation. Hum Reprod Update 12: 731-746, 2006.

30. Altmäe S, Koel M, Võsa U, Adler P, Suhorutšenko M, Laisk-Podar T, Kukushkina V, Saare M, Velthut-Meikas A, Krjutškov K, et al: Meta-signature of human endometrial receptivity: A meta-analysis and validation study of transcriptomic biomarkers. Sci Rep 7: 10077, 2017.

31. Díaz-Gimeno P, Horcajadas JA, Martínez-Conejero JA, Esteban FJ, Alamá P, Pellicer A and Simón C: A genomic diagnostic tool for human endometrial receptivity based on the transcriptomic signature. Fertil Steril 95: 50-60, 60 e1-15, 2011.

32. Julkunen M, Koistinen R, Sjöberg J, Rutanen EM, Wahlström T and Seppälä M: Secretory endometrium synthesizes placental protein 14. Endocrinology 118: 1782-1786, 1986.

33. Chan C, Virtanen C, Winegarden NA, Colgan TJ, Brown TJ and Greenblatt EM: Discovery of biomarkers of endometrial receptivity through a minimally invasive approach: A validation study with implications for assisted reproduction. Fertil Steril 100 810-817, 2013

34. Burmenskaya OV, Bozhenko VK, Smolnikova VY, Kalinina EA, Korneeva IE, Donnikov AE, Beyk EP, Naumov VA, Aleksandrova NV, Borovikov PI and Trofimov DY: Transcription profile analysis of the endometrium revealed molecular markers of the personalized 'window of implantation' during in vitro fertilization. Gynecol Endocrinol 33 (Supp1): S22-S27, 2017.

35. Zhang D, Sun C, Ma C, Dai H and Zhang W: Data mining of spatial-temporal expression of genes in the human endometrium during the window of implantation. Reprod Sci 19: 1085-1098, 2012.

36. Zhang Z, Zhang L, Zhang L, Jia L, Wang P and Gao Y: Association of Wnt 2 and sFRP4 expression in the third trimester placenta in women with severe preeclampsia. Reprod Sci 20: 981-989, 2013

37. Kodithuwakku SP, Ng PY, Liu Y, Ng EH, Yeung WS, Ho PC and Lee KF: Hormonal regulation of endometrial olfactomedin expression and its suppressive effect on spheroid attachment onto endometrial epithelial cells. Hum Reprod 26: 167-175, 2011

38. So KH, Kodithuwakku SP, Kottawatta KS, Li RH, Chiu PC, Cheung AN, Ng EH, Yeung WS and Lee KF: Human chorionic gonadotropin stimulates spheroid attachment on fallopian tube epithelial cells through the mitogen-activated protein kinase pathway and down-regulation of olfactomedin-1. Fertil Steril 104: 474-482, 2015

39. Tan Y, Xiao D, Xu Y and Wang C: Long non-coding RNA DLX6-AS1 is upregulated in preeclampsia and modulates migration and invasion of trophoblasts through the miR-376c/GADD45A axis. Exp Cell Res 370: 718-724, 2018.

40. Li R, He J, Chen X, Ding Y, Wang Y, Long C, Shen L and Liu X: Mmu-miR-193 is involved in embryo implantation in mouse uterus by regulating GRB7 gene expression. Reprod Sci 21: 733-742, 2014

41. Balaguer N, Moreno I, Herrero M, Gonzáléz-Monfort M, Vilella F and Simón C: MicroRNA-30d deficiency during preconception affects endometrial receptivity by decreasing implantation rates and impairing fetal growth. Am J Obstet Gynecol 221: 46 e1-46 e16, 2019.

42. Ren JW, Li ZJ and Tu C: MiR-135 post-transcriptionally regulates FOXO1 expression and promotes cell proliferation in human malignant melanoma cells. Int J Clin Exp Pathol 8: 6356-6366, 2015 .

43. Singh H and Aplin JD: Adhesion molecules in endometrial epithelium: Tissue integrity and embryo implantation. J Anat 215: 3-13, 2009 .

44. Kusama K, Yoshie M, Tamura K, Daikoku T, Takarada T and Tachikawa E: Possible roles of the cAMP-mediators EPAC and RAP1 in decidualization of rat uterus. Reproduction 147 897-906, 2014

45. Seo JS, Rhie A, Kim J, Lee S, Sohn MH, Kim CU, Hastie A, Cao H, Yun JY, Kim J, et al: De novo assembly and phasing of a Korean human genome. Nature 538: 243-247, 2016.

46. Galkin F, Mamoshina P, Aliper A, de Magalhaes JP, Gladyshev VN and Zhavoronkov A: Biohorology and biomarkers of aging: Current state-of-the-art, challenges and opportunities. Ageing Res Rev 60: 101050, 2020.

47. Gibson DA, Simitsidellis I, Cousins FL, Critchley HO and Saunders PT: Intracrine androgens enhance decidualization and modulate expression of human endometrial receptivity genes. Sci Rep 6: 19970, 2016.
48. Henriquez S, Tapia A, Quezada M, Vargas M, Cardenas H, Rios M, Salvatierra AM, Croxatto H, Orihuela P, Zegers-Hochschild F, et al: Deficient expression of monoamine oxidase A in the endometrium is associated with implantation failure in women participating as recipients in oocyte donation. Mol Hum Reprod 12: 749-754, 2006.

49. Tapia A, Gangi LM, Zegers-Hochschild F, Balmaceda J, Pommer R, Trejo L, Pacheco IM, Salvatierra AM, Henríquez S, Quezada M, et al: Differences in the endometrial transcript profile during the receptive period between women who were refractory to implantation and those who achieved pregnancy. Hum Reprod 23: 340-351, 2008.

50. Li DD, Yin YH, Wu JY, Yang ZQ, Cao H, Zhang QL, Guo B and Yue ZP: Effects of Idol on mouse decidualization. Mol Biol (Mosk) 49: 649-657, 2015 (In Russian).

51. Christian M, Zhang X, Schneider-Merck T, Unterman TG, Gellersen B, White JO and Brosens JJ: Cyclic AMP-induced forkhead transcription factor, FKHR, cooperates with CCAAT/enhancer-binding protein beta in differentiating human endometrial stromal cells. J Biol Chem 277: 20825-20832, 2002.

52. Kajihara T, Jones M, Fusi L, Takano M, Feroze-Zaidi F, Pirianov G, Mehmet H, Ishihara O, Higham JM, Lam EW and Brosens JJ: Differential expression of FOXO1 and FOXO3a confers resistance to oxidative cell death upon endometrial decidualization. Mol Endocrinol 20: 2444-2455, 2006.

53. Labied S, Kajihara T, Madureira PA, Fusi L, Jones MC, Higham JM, Varshochi R, Francis JM, Zoumpoulidou G, Essafi A, et al: Progestins regulate the expression and activity of the forkhead transcription factor FOXO1 in differentiating human endometrium. Mol Endocrinol 20: 35-44, 2006.

54. Lehtinen MK, Yuan Z, Boag PR, Yang Y, Villén J, Becker EB, DiBacco S, de la Iglesia N, Gygi S, Blackwell TK and Bonni A: A conserved MST-FOXO signaling pathway mediates oxidative-stress responses and extends life Span. Cell 125: 987-1001, 2006.

55. Rached MT, Kode A, Xu L, Yoshikawa Y, Paik JH, Depinho RA and Kousteni S: FoxO1 is a positive regulator of bone formation by favoring protein synthesis and resistance to oxidative stress in osteoblasts. Cell Metab 11: 147-160, 2010.

56. Gellersen B, Brosens IA and Brosens JJ: Decidualization of the human endometrium: Mechanisms, functions, and clinical perspectives. Semin Reprod Med 25: 445-453, 2007.

57. Liu Y, Kodithuwakku SP, Ng PY, Chai J, Ng EH, Yeung WS, Ho PC and Lee KF: Excessive ovarian stimulation up-regulates the Wnt-signaling molecule DKK1 in human endometrium and may affect implantation: An in vitro co-culture study. Hum Reprod 25: 479-490, 2010.

58. Daikoku T, Cha J, Sun X, Tranguch S, Xie H, Fujita T, Hirota Y, Lydon J, DeMayo F, Maxson R and Dey SK: Conditional deletion of Msx homeobox genes in the uterus inhibits blastocyst implantation by altering uterine receptivity. Dev Cell 21: 1014-1025, 2011.

59. Wang $X$ and $Y u$ Q: Endometriosis-related ceRNA network to identify predictive biomarkers of endometrial receptivity. Epigenomics 11: 147-167, 2019.

60. Xu H, Zhou M, Cao Y, Zhang D, Han M, Gao X, Xu B and Zhang A: Genome-wide analysis of long noncoding RNAs, microRNAs, and mRNAs forming a competing endogenous RNA network in repeated implantation failure. Gene 720 : 144056, 2019.

61. Fang C, Xu L, He W, Dai J and Sun F: Long noncoding RNA DLX6-AS1 promotes cell growth and invasiveness in bladder cancer via modulating the miR-223-HSP90B1 axis. Cell Cycle 18: 3288-3299, 2019.

62. Wang X, Lin Y and Liu J: Long noncoding RNA DLX6AS1 promotes proliferation by acting as a ceRNA targeting miR199a in cervical cancer. Mol Med Rep 19: 1248-1255, 2019.

63. Guo Q, Sun H, Zheng K, Yin S and Niu J: Long non-coding RNA DLX6-AS1/miR-141-3p axis regulates osteosarcoma proliferation, migration and invasion through regulating Rab10. RSC Adv 9: 33823-33833, 2019.

64. Hawkins SM, Creighton CJ, Han DY, Zariff A, Anderson ML, Gunaratne PH and Matzuk MM: Functional microRNA involved in endometriosis. Mol Endocrinol 25: 821-832, 2011.

65. Liu X, Gao R, Chen X, Zhang H, Zheng A, Yang D, Ding Y, Wang Y and He J: Possible roles of mmu-miR-141 in the endometrium of mice in early pregnancy following embryo implantation. PLoS One 8: e67382, 2013 
66. Jimenez PT, Mainigi MA, Word RA, Kraus WL and Mendelson CR: miR-200 regulates endometrial development during early pregnancy. Mol Endocrinol 30: 977-987, 2016.

67. Wu XM, Shao XQ, Meng XX, Zhang XN, Zhu L, Liu SX, Lin J and Xiao HS: Genome-wide analysis of microRNA and mRNA expression signatures in hydroxycamptothecin-resistant gastric cancer cells. Acta Pharmacol Sin 32: 259-269, 2011.

68. Kottawatta KS, So KH, Kodithuwakku SP, Ng EH, Yeung WS and Lee KF: MicroRNA-212 regulates the expression of olfactomedin 1 and $\mathrm{C}$-terminal binding protein 1 in human endometrial epithelial cells to enhance spheroid attachment in vitro. Biol Reprod 93: 109, 2015.

69. Franco-Murillo Y, Miranda-Rodríguez JA, Rendón-Huerta E, Montaño LF, Cornejo GV, Gómez LP, Valdez-Morales FJ, Gonzalez-Sanchez I and Cerbón M: Unremitting cell proliferation in the secretory phase of eutopic endometriosis: Involvement of pAkt and pGSK3ß. Reprod Sci 22: 502-510, 2015.

70. Li W, Ma S, Bai X, Pan W, Ai L and Tan W: Long noncoding RNA WDFY3-AS2 suppresses tumor progression by acting as a competing endogenous RNA of microRNA-18a in ovarian cancer. J Cell Physiol 235: 1141-1154, 2020.

71. Zhang Q, Guan F, Fan T, Li S, Ma S, Zhang Y, Guo W and Liu H: LncRNA WDFY3-AS2 suppresses proliferation and invasion in oesophageal squamous cell carcinoma by regulating miR-2355-5p/SOCS2 axis. J Cell Mol Med 24: 8206-8220, 2020

72. Wu F, Zhao Z, Chai R, Liu Y, Wang K, Wang Z, Li G, Huang R, Jiang $\mathrm{H}$ and Zhang $\mathrm{K}$ : Expression profile analysis of antisense long non-coding RNA identifies WDFY3-AS2 as a prognostic biomarker in diffuse glioma. Cancer Cell Int 18: 107, 2018.

73. Wang J, Zhang L, Jiang W, Zhang R, Zhang B, Silayiding A and Duan X: MicroRNA-135a promotes proliferation, migration, invasion and induces chemoresistance of endometrial cancer cells. Eur J Obstet Gynecol Reprod Biol X 5: 100103, 2019.

74. Zeng YB, Liang XH, Zhang GX, Jiang N, Zhang T, Huang JY, Zhang L and Zeng XC: miRNA-135a promotes hepatocellular carcinoma cell migration and invasion by targeting forkhead box O1. Cancer Cell Int 16: 63, 2016.

75. Xiao LJ, Yuan JX, Song XX, Li YC, Hu ZY and Liu YX: Expression and regulation of stanniocalcin 1 and 2 in rat uterus during embryo implantation and decidualization. Reproduction 131: 1137-1149, 2006.
76. Aghajanova L, Altmae S, Kasvandik S, Salumets A, Stavreus-Evers A and Giudice LC: Stanniocalcin-1 expression in normal human endometrium and dysregulation in endometriosis. Fertil Steril 106: 681-691 e1, 2016.

77. Estella C, Herrer I, Moreno-Moya JM, Quiñonero A, Martínez S, Pellicer A and Simón C: miRNA signature and Dicer requirement during human endometrial stromal decidualization in vitro. PLoS One 7: e41080, 2012

78. Zhang L, Quan H, Wang S, Li X and Che X: MiR-183 promotes growth of non-small cell lung cancer cells through FoxO1 inhibition. Tumour Biol 36: 8121-8126, 2015.

79. Akbar R, Ullah K, Rahman TU, Cheng Y, Pang HY, Jin LY, Wang QJ, Huang HF and Sheng JZ: miR-183-5p regulates uterine receptivity and enhances embryo implantation. J Mol Endocrinol 64: 43-52, 2020.

80. Gao JM, Huang LZ, Huang ZG and He RQ: Clinical value and potential pathways of miR-183-5p in bladder cancer: A study based on miRNA-seq data and bioinformatics analysis. Oncol Lett 15: 5056-5070, 2018.

81. Li Y, Yan J, Wang Y, Wang C, Zhang C and Li G: LINC00240 promotes gastric cancer cell proliferation, migration and EMT via the miR-124-3p/DNMT3B axis. Cell Biochem Funct 38: $1079-1088,2020$

82. Zhang Y, Li X, Zhang J and Liang H: Natural killer T cell cytotoxic activity in cervical cancer is facilitated by the LINC00240/microRNA-124-3p/STAT3/MICA axis. Cancer Lett 474: 63-73, 2020.

83. Myatt SS, Wang J, Monteiro LJ, Christian M, Ho KK, Fusi L, Dina RE, Brosens JJ, Ghaem-Maghami S and Lam EW: Definition of microRNAs that repress expression of the tumor suppressor gene FOXO1 in endometrial cancer. Cancer Res 70: 367-377, 2010.

84. Meng N, Yang Q, He Y, Gu WW, Gu Y, Zhen XX, Wang J, Zhang X, Sun ZG and Wang J: Decreased NDRG1 expression is associated with pregnancy loss in mice and attenuates the in vitro decidualization of endometrial stromal cells. Mol Reprod Dev 86: 1210-1223, 2019.

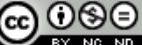

This work is licensed under a Creative Commons

Attribution-NonCommercial-NoDerivatives 4.0

International (CC BY-NC-ND 4.0) License. 